\title{
ELEMENTARY AND MULTIELEMENTARY REPRESENTATIONS OF VECTROIDS
}

\author{
K. I. Belousov, L. A. Nazarova, A. V. Roiter, and V. V. Sergeichuk \\ UDC 519.1
}

\section{To the memory of Maurice Auslender}

\begin{abstract}
We prove that every finitely represented vectroid is determined, up to an isomorphism, by its completed biordered set. Elementary and multielementary representations of such vectroids (which play a central role for biinvolutive posets) are described.
\end{abstract}

\section{Introduction}

Denote by $k$ a fixed algebraically closed field and by $\bmod k$ the category of finite-dimensional right vector spaces over $k$. The symbol of a linear map is written to the right.

$A$ vectroid $\mathcal{V}$ (over the field $k$ ) is a small (the class of objects is a set) subcategory of the category $\bmod k$ which is a spectroid in the sense of [1], i.e., satisfies the following conditions:

(i) For each pair of objects $X, Y \in \mathcal{V}$, the set $\mathcal{V}(X, Y)$ of morphisms is a linear subspace in $\bmod k(X, Y)$;

(ii) For each $X \in \mathcal{V}$, the ring $\mathcal{V}(X, X)$ contains exactly two idempotents $\left(0_{X} \neq 1_{X}\right)$;

(iii) $\mathcal{V}$ does not contain isomorphic objects.

The value $\sup \{\operatorname{dim} X, X \in \mathcal{V}\}$ is called the dimension $\operatorname{dim} \mathcal{V}$ of $\mathcal{V}$.

Each vectroid $\mathcal{V}$ defines a category (an aggregate in the sense of [1]) $\oplus \mathcal{V} \subset \bmod k$ whose objects are all finite direct sums $X_{1} \oplus \ldots \oplus X_{m}\left(X_{i} \in \mathcal{V}, m \geq 0\right)$. The category $\oplus \mathcal{V}$ (as well as any subcategory in $\left.\bmod k\right)$ can be regarded as a faithful module over itself [1].

A triple $(U, f, X)$ consisting of the spaces $U \in \bmod k$ and $X \in \oplus \mathcal{V}$ and a linear map $f: U \rightarrow X$ is called a representation of $\mathcal{V}([1], 4.1 ;[2])$. The morphism $(U, f, X) \rightarrow\left(U^{\prime}, f^{\prime}, X^{\prime}\right)$ is a pair $(\varphi, \xi)$ that consists of a linear map $\varphi: U \rightarrow U^{\prime}$ and a morphism $\xi: X \rightarrow X^{\prime}$ of the category $\oplus \mathcal{V}$ such that $\varphi f^{\prime}=f \xi$. Representations form the aggregate denoted by $\operatorname{Rep} \mathcal{V}$. A vectroid is called finitely represented if Rep $\mathcal{V}$ has finitely many indecomposable nonisomorphic objects.

Within the notation of $([1], 4.1)$, the category $\operatorname{Rep} \mathcal{V}$ coincides with the category $(\oplus \mathcal{V})^{k}$. In some cases, we shall consider the category $M^{k}$ of representations of an arbitrary module $M$ (not necessarily faithful) over an aggregate (see Appendix at the end of Introduction).

If $\operatorname{dim} \mathcal{V}=1$, then $\mathcal{V}$ is completely determined by the following partial ordering of the set $\mathrm{Ob} \mathcal{V}: X \leq Y$ if $\mathcal{V}(X, Y) \neq 0$. The category $\operatorname{Rep} \mathcal{V}$ can be naturally identified with the category of representations of this poset ([1] $4.1 ;[5])$. The criterion of finite representability of posets was obtained in [6].

On the other hand, it was proved in $([1], 4.2,4.3)$ and $([3], 9.1,9.4)$ that the category $\bmod \Lambda$ of representations of an arbitrary finite-dimensional algebra $\Lambda$ over $k$ coincides with the category of representations of a certain vectroid $\mathcal{V}$ in the following sense: There exists an injective indecomposable $\Lambda$-module $P$ such that the category of all $\Lambda$-modules that do not contain $P$ as a direct summand is epivalent to $\operatorname{Rep} \mathcal{V}$.

Institute of Mathematics, Ukrainian Academy of Sciences, Kiev. Translated from Ukrainskii Matematicheskii Zhurnal, Vol. 47, No. 11, pp. 1451-1477, November, 1995. Original article submitted December 29, 1994. 
Thus, the theory of representations of vectroids can be regarded as a generalization of the theory of representations of posets and the theory of representations of finite-dimensional algebras.

We define the radical of a vectroid $\mathcal{V}$ as the ideal of $\mathcal{V}$ generated by the spaces $\operatorname{Rad}_{\mathcal{V}}(X, Y)$ of uninvertible morphisms from $\mathcal{V}(X, Y)$ for all $X, Y \in \mathcal{V}$. It is obvious that $\mathcal{V}(X, Y)=\operatorname{Rad}_{\mathcal{V}}(X, Y)$ for $X \neq Y$ and $\mathcal{V}(X, X)=k 1_{X} \oplus \operatorname{Rad}_{\mathcal{V}}(X, X)$.

The set $\left\{\left(n_{i}^{X}\right),\left(f_{l}^{X Y}\right)\right\}$ that consists of the bases $\left(n_{1}^{X}, n_{2}^{X}, \ldots, n_{\operatorname{dim}(X)}^{X}\right)$ of spaces $X \in \mathcal{V}$ and the bases $\left(f_{1}^{X Y}, f_{2}^{X Y}, \ldots\right)$ of spaces $\operatorname{Rad}_{\mathcal{V}}(X, Y)$ is called the basis of the vectroid $\mathcal{V}$. The maximal rank of the linear maps $f_{l}^{X Y}$ is called the rank of the basis. The basis $\left(n_{1}^{X}, n_{2}^{X}, \ldots, n_{\operatorname{dim}(X)}^{X}\right)$ of an object $X \in \mathcal{V}$ is called triangular if the family $\left\{\overline{\left(n_{i}^{X}\right)}, i=\overline{1, \operatorname{dim} X} \mid \overline{\left(n_{i}^{X}\right)} \neq 0\right\}$ is linearly independent for any $j \in \mathbb{N}$, where the bar means the transition to the factor space $X / X \operatorname{Rad}_{\mathcal{V}}^{j}(X, X)$. The basis $\left\{\left(n_{i}^{X}\right),\left(f_{l}^{X Y}\right)\right\}$ of $\mathcal{V}$ is called triangular if each basis $\left(n_{i}^{X}\right), X \in \mathcal{V}$, is triangular. A basis is scalarly multiplicative if the element $n_{i}^{X} f_{l}^{X Y}$ is equal to $\lambda n_{p}^{Y}, \lambda \in k$, for all $n_{i}^{X}, f_{l}^{X Y}$, and it follows from the relations $n_{i}^{X} f_{l}^{X Y}=\lambda n_{p}^{Y}$ and $n_{j}^{X} f_{l}^{X Y}=\mu n_{p}^{Y}, \lambda$, $\mu \in k^{*}$, that $i=j$. A scalarly multiplicative basis is called multiplicative if each element $n_{i}^{X} f_{l}^{X Y}$ is equal to either 0 or $n_{p}^{Y}([1], 4.10)$. Every finitely represented vectroid has a multiplicative basis whose rank does not exceed two [4].

A vectroid $\mathcal{V}$ is called a chain vectroid if, for every $X \in \mathcal{V}$, submodules of the module $X_{\mathcal{V}(X X)}$ are linearly ordered with respect to the inclusions

$$
X=X_{1} \underset{\ddagger}{\supset} X_{2} \underset{\ddagger}{\supset} \underset{\ddagger}{\supset} X_{\mathrm{dim} X} \underset{\ddagger}{\supset} 0
$$

In this case, all these submodules are cyclic, $X_{i}=m_{i}^{X} \mathcal{V}(X, X)$, and $m_{1}^{X}, \ldots, m_{\operatorname{dim} X}^{X}$ is a triangular basis of $X \in \mathcal{V}$ (see Lemma 1).

It is known that if $\mathcal{V}$ is a finitely represented vectroid, then $\mathcal{V}$ is a chain vectroid and $\operatorname{dim} \mathcal{V} \leq 3$ ([1], 4.7 and 4.8).

For an arbitrary chain vectroid $\mathcal{V}$, we construct the poset

$$
S(\mathcal{V})=\bigcup_{X \in \mathcal{V}}\left\{X_{1}, X_{2}, \ldots, X_{\operatorname{dim} X}\right\}
$$

setting $X_{i} \leq Y_{j}$ if $m_{i}^{X} \varphi=m_{j}^{Y}$ for some $\varphi \in \mathcal{V}(X, Y)$.

The number $\operatorname{def}(\mathcal{V})=\sup \{\operatorname{def}(X, Y) \mid X, Y \in \mathrm{Ob} \mathcal{V}\}$, where

$$
\operatorname{def}(X, Y)=\left|\left\{\left(X_{i}, Y_{j}\right) \mid X_{i}<Y_{j}\right\}\right|-\operatorname{dim} \operatorname{Rad}(X, Y)
$$

is called the defect of $\mathcal{V}$. According to [4], we have def $\mathcal{V} \leq 1$ for all finitely represented vectroids $\mathcal{V}$ (see Sec. 2).

If def $\mathcal{V}=0$, then $\mathcal{V}$ has a multiplicative basis of rank one and the category Rep $\mathcal{V}$ coincides with the category of representations of a weakly completed poset $S(\mathcal{V})$ (see Sec. 1). The criterion of finite representability of weakly completed posets and the classification of their indecomposable representations (in the case of finite representability) are given in [9] (see also [10]).

Let $\operatorname{def} \mathcal{V}=1$ and $\operatorname{dim} \mathcal{V}=2$. In this case, representations of vectroids $\mathcal{V}$ are identified with representations of a certain poset $S(\mathcal{V})$ with additional structure (the structure of a biinvolutive poset). In this case, $\mathcal{V}$ is finitely represented if and only if a certain poset $\operatorname{St}(S(\mathcal{V}))$ constructed for a biinvolutive poset $S([1], 5.8)$ is finitely represented. This criterion was formulated in [1] and proved in $[7,8]$. 
Thus, the problem of finite representability remains open only for three-dimensional (chain) vectroids with defect one.

In Sec. 1, we associate every chain vectroid $\mathcal{V}$ with a completed biordered set $S(\mathcal{V})$. In Sec. 2, we show that a finitely represented vectroid is uniquely determined by its completed biordered set. In Sec. 5 , for every chain vectroid $\mathcal{V}$ with def $\mathcal{V} \leq 1$, we construct a poset $C(\mathcal{V})$, which is a generalization of the poset $\operatorname{St}(S)([1], 5.8)$. In this connection, we introduce elementary and multielementary representations of vectroids.

\section{Appendix. Modules over Aggregates}

Let $\mathcal{M}$ be a category of pairs $(A, M)$, where $A$ is an aggregate and $M$ is a pointwise finite (see [1], 3.1, 2.2, and 3.6) right module over $A$. A morphism from $(A, M)$ to $\left(A^{\prime}, M^{\prime}\right)$ is a pair $(F, \Phi)$, where $F: A \rightarrow A^{\prime}$ is a functor, $\Phi: M \rightarrow F^{*} M^{\prime}$ is a morphism of $A$-modules, and $F^{*} M^{\prime}$ is a restriction of $M^{\prime}$ along $F$.

On the basis of the module $(A, M) \in \mathcal{M}$, we construct a derivative module $\operatorname{Der}(A, M)=\left(\operatorname{Rep} M, E_{M}\right)$. Here, $\operatorname{Rep} M$ is an aggregate consisting of triples $(V, f, X)$ with $V \in \bmod k, X \in A$, and $f \in \bmod k(V, M(X))$; morphisms from $(V, f, X)$ to $\left(V^{\prime}, f^{\prime}, X^{\prime}\right)$ are pairs $(\varphi, \xi)$, where $\varphi \in \bmod k\left(V, V^{\prime}\right)$ and $\xi \in A\left(X, X^{\prime}\right)$, such that $f \circ M \xi=\varphi \circ f^{\prime}$ (cf. [1], 4.1, where $\operatorname{Rep} M$ is denoted by $M^{k}$ ); $E_{M}$ is a module over Rep $M$ obtained from the module $(A, M)$ by the restriction along the functor $T: \operatorname{Rep} M \rightarrow A, T(V, f, X)=X$.

Moreover, Der can be continued up to the functor Der: $\mathcal{M} \rightarrow \mathcal{M}$ that transforms the morphism $(F, \Phi):$ $(A, M) \rightarrow\left(A^{\prime}, M^{\prime}\right)$ into the morphism

$$
\operatorname{Der}(F, \Phi)=(G, \Psi):\left(\operatorname{Rep} M, E_{M}\right) \rightarrow\left(\operatorname{Rep} M^{\prime}, E_{M}\right)
$$

where $G(V, f, X)=(V, f \circ \Phi(X), F(X)), \Psi(V, f, X)=\Phi(X)$ (here, $\left.\Phi(X): M(X) \rightarrow M^{\prime}(F X)\right)$.

On the basis of an arbitrary vectroid $\mathcal{V}$, we naturally construct a module $\mathrm{M} \mathcal{V}=\left(\oplus \mathcal{V}, M_{\mathcal{V}}\right) \in \mathcal{M}$, where $\oplus \mathcal{V}$ is the aggregate of all direct sums of objects from $\mathcal{V}$, and $M_{\mathcal{V}}$ is a module over $\oplus \mathcal{V}, M_{\mathcal{V}}(X)=X$ (recall that every object $X \in \oplus \mathcal{V}$ can be regarded as a vector space). The obtained module is faithful (i.e., $M_{\mathcal{V}} \xi \neq 0$ if $\left.0 \neq \xi \in \oplus \mathcal{V}\left(X, X^{\prime}\right)\right)$.

In what follows, we omit the indication of the map $\mathrm{M}$, i.e., we write $\mathcal{M}\left(V, V^{\prime}\right)$ instead of $\mathcal{M}\left(\mathrm{M} V, \mathrm{M} V^{\prime}\right)$, Der $\mathcal{V}$ instead of $\operatorname{Der}(\mathrm{M} \mathcal{V})$, etc. Note that $\operatorname{Rep} \mathrm{M} \mathcal{V}$ coincides with $\operatorname{Rep} \mathcal{V}$, and the vectroids $\mathcal{V}$ and $\mathcal{V}^{\prime}$ are isomorphic if and only if the modules $\mathrm{M} \mathcal{V}$ and $\mathrm{M} \mathcal{V}^{\prime \prime}$ are isomorphic.

Remark I. Some authors (e.g., Nazarova and Roiter [2]) studied the category of subspaces $U_{\mathcal{V}}$ of the aggregate $\oplus \mathcal{V}$, where $\mathcal{V}$ is an arbitrary vectroid. Objects of this category are subspaces of the spaces $X \in \oplus \mathcal{V}$. The set of morphisms $U_{\mathcal{U}}(V, W)$ consists of all $\varphi \in \oplus \mathcal{V}(X, Y)$ such that $V \varphi \subset W$, where $V \subset X, W \subset Y$. Suppose that ${ }^{i} \operatorname{Rep} \mathcal{V} \subset \operatorname{Rep} \mathcal{V}$ is a complete subcategory consisting of representations $(V, f, X) \in \operatorname{Rep} \mathcal{V}$ such that $f$ is a monomorphism. The spectroid of the aggregate $\operatorname{Rep} \mathcal{V}$ contains a full subspectroid, which is "equal" to the spectroid of the aggregate ${ }^{i} \operatorname{Rep} \mathcal{V}$, and exactly one more object, namely, $(k, 0,0)$. It is easy to see that the categories $U_{\mathcal{V}}$ and ${ }^{i} \operatorname{Rep} \mathcal{V}$ are equivalent.

\section{Biordered Sets}

Let $\alpha$ be a binary relation on a set $Z$. We define

$$
A^{\alpha}(b)=\{y \in A \mid y \alpha b\}, \quad A^{\alpha}(B)=\bigcap_{b \in B} A^{\alpha}(b)
$$


for $b \in Z$ and $A, B \subset Z$.

We write $A \alpha B$ if $a \alpha b$ for all $a \in A$ and $b \in B$.

For a partial order relation $\leq$ on the set $Z$ and for $a, b \in Z$, we write $a \nless b$ if $a \leq b$ and $b \leq a$.

Denote by $\tilde{\alpha}$ the reflexive closure of a relation $\alpha$, i.e., a relation on $Z$ such that $z \tilde{\alpha} z$ for all $z \in Z$, and $z \tilde{\alpha} y$ for $y \neq z$ if and only if $z \alpha y$. A relation $\alpha$ is called a pseudoequivalence if $\alpha$ is antireflexive and $\tilde{\alpha}$ is an equivalence. In the present paper, all pseudoequivalences and the corresponding equivalence relations are denoted by $\sim$ and $\approx$, respectively.

A set $S$ is called a biordered set [8] if it is equipped with the partial order relation $\leq$ and the relation $\triangleleft$ that satisfy the following conditions:

(a) if $a \triangleleft b$, then $a \leq b$;

(b) if $a \triangleleft b \leq c$ or $a \leq b \triangleleft c$, then $a \triangleleft c$.

Note that the relation $\triangleleft$ is transitive and antisymmetric but, in general, not reflexive (if $\triangleleft$ is reflexive, it coincides with $\leq$ ).

Remark 2. If Cat $(S, \leq)$ is a poset $(S, \leq)$ considered as a category (see [1], 2.1, Example 5), then $\triangleleft=$ $\{(Y \mid X) \mid X \triangleleft Y\}$ is an ideal in Cat $(S, \leq)$. Conversely, every ideal in this category determines a biordered set.

A biordered set with a given equivalence relation $\approx$ is called a completed biordered set. A completed biordered set $(S, \leq, \triangleleft, \approx)$ is called locally finite if all equivalence classes $S^{\approx}(s), s \in S$, are finite.

A poset $(S, \leq)$ with the equivalence relation $\approx$ is called a weakly completed poset. If $S=(S, \leq, \triangleleft, \approx)$ is a completed biordered set, then $S^{w}=(S, \leq, \approx)$ is a weakly completed poset obtained by weakening the structure on $S$. In some cases, it is convenient to regard a weakly completed poset $(S, \leq, \approx)$ as a completed biordered set in which the relations $\leq$ and $\triangleleft$ coincide.

Let $S$ be a completed biordered set. We denote the number of elements that are equivalent to $a \in S$ by $d(a)$ and set $d(S)=\sup \{d(a) \mid a \in S\}$. We say that a pair $(a, b)$ is an edge and write $a \Rightarrow b$ if $a<b$ and $a \pitchfork b$. By the definition of a biordered set, if $a \Rightarrow b$ and $a<x<b$, then $a \Rightarrow x \Rightarrow b$. Two edges $\alpha: a \Rightarrow b$ and $\alpha^{\prime}$ : $a^{\prime} \Rightarrow b^{\prime}$ are called equivalent and denoted by $\alpha \approx \alpha^{\prime}$ (or $\alpha \sim \alpha^{\prime}$ for $\alpha \neq \alpha^{\prime}$ ) if $a \approx a^{\prime}$ and $b \approx b^{\prime}$. The number of edges equivalent to $a \Rightarrow b$ is denoted by ed $(a, b)$. An edge $a \Rightarrow b$ is called maximal if $x \leq a<b \leq y$ and $x \Rightarrow y$ imply that $x=a$ and $y=b$. An edge $a \Rightarrow b$ is called short if there is no $x$ such that $a<x<b$. A pair of equivalent edges $(a \Rightarrow b) \sim\left(a^{\prime} \Rightarrow b^{\prime}\right)$ is called short if there is no $x \sim x^{\prime}$ such that $a<x<b$ and $a^{\prime}<$ $x^{\prime}<b^{\prime}$ (see Example 1, (c) below).

Let $\mathcal{V}$ be a chain vectroid. We introduce the structure of a completed biordered set on the poset $S(\mathcal{V})$ as follows: Let $X_{i}=m_{i}^{X} \mathcal{V}(X, X)$ and $Y_{j}=m_{j}^{Y} \mathcal{V}(Y, Y) \in S(\mathcal{V})$. We set $X_{i} \approx Y_{j}$ if $X=Y$, and $X_{i} \triangleleft Y_{j}$ if there exists a linear map $\varphi \in \mathcal{V}(X, Y)$ of rank one such that $m_{i}^{X} \varphi=m_{j}^{Y}$.

Remark 3. By analogy, we can define a completed biordered set $S(\mathcal{V})$ (of cyclic submodules) if $\mathcal{V}$ is not a chain vectroid. However, it can be not locally finite. Note that a vectroid $\mathcal{V}$ is a chain vectroid if and only if the module $X_{\mathcal{V}(X, X)}$ contains only a finite number of cyclic submodules for any $X \in \mathcal{V}$.

Let $X=\oplus_{i} k m_{i}^{X}$ and $Y=\oplus_{j} k m_{j}^{Y}$ be two objects of a vectroid $\mathcal{V}$. We define a linear map $e_{i j}^{X Y}: X \rightarrow Y$ by setting $m_{i}^{X} e_{i j}^{X Y}=m_{j}^{Y}$ and $m_{l}^{X} e_{i j}^{X Y}=0$ for $l \neq i$.

Example 1. (a) Consider the vectroid $\mathcal{V}$ with two objects 


$$
X=\bigoplus_{i=1}^{3} k m_{i}^{X} \quad \text { and } \quad Y=\bigoplus_{i=1}^{3} k m_{i}^{Y}
$$

and the following spaces of morphisms:

$$
\begin{gathered}
\mathcal{V}_{1}(X, X)=k 1_{X} \oplus \underset{i<j}{\oplus} k e_{i j}^{X X}, \quad \mathcal{V}_{1}(Y, Y)=k 1_{Y} \oplus \underset{i<j}{\oplus} k e_{i j}^{Y Y}, \\
\mathcal{V}_{1}(X, Y)=k\left(e_{11}^{X Y}+e_{22}^{X Y}\right) \oplus k\left(e_{11}^{X Y}+e_{33}^{X Y}\right) \oplus \underset{i<j}{\oplus} k e_{i j}^{X Y}, \\
\mathcal{V}_{1}(Y, X)=\underset{i<j}{\oplus} k e_{i j}^{Y X} .
\end{gathered}
$$

Obviously, $\operatorname{dim}\left(\mathcal{V}_{1}\right)=3, \operatorname{def}\left(\mathcal{V}_{1}\right)=1$, and

$$
\begin{aligned}
x_{1} \circ & \Longleftrightarrow \circ Y_{1} \\
S\left(\mathcal{V}_{1}\right)=x_{2} \circ & \stackrel{\Longleftrightarrow}{\Longleftrightarrow} \circ Y_{2} \\
x_{3} \circ & \Longleftrightarrow \circ Y_{3}
\end{aligned}
$$

( $a \rightarrow b$ means that $a \triangleleft b$ and there is no $x \in S$ such that $a \triangleleft x<b$ or $a<x \triangleleft b$ ).

(b) Consider the vectroid $\mathcal{V}_{2}$ that differs from $\mathcal{V}_{1}$ only by the following space of morphisms:

$$
\mathcal{V}_{2}(X, Y)=k\left(e_{11}^{X Y}+e_{22}^{X Y}+e_{33}^{X Y}\right) \oplus \underset{i<j}{\oplus} k e_{i j}^{X Y}
$$

Clearly, $\operatorname{dim}\left(\mathcal{V}_{2}\right)=3, \operatorname{def}\left(\mathcal{V}_{2}\right)=2$, and $S\left(\mathcal{V}_{2}\right)=S\left(\mathcal{V}_{1}\right)$

(c) We construct the vectroid $\mathcal{V}_{3}$ by completing $\mathcal{V}_{1}^{\prime}$ with the objects $Z=k m_{1}^{Z} \oplus k m_{2}^{Z}$ and $T=k m_{1}^{T} \oplus k m_{2}^{T}$ with the following spaces of morphisms:

$$
\begin{gathered}
\mathcal{V}_{3}(Z, Z)=k 1_{Z} \oplus k e_{12}^{Z Z}, \quad \mathcal{V}_{3}(T, T)=k 1_{T} \oplus k e_{12}^{T T}, \\
\mathcal{V}_{3}(X, Z)=k\left(e_{11}^{X Z}+e_{22}^{X Z}\right), \quad \mathcal{V}_{3}(Z, Y)=k\left(e_{11}^{Z Y}+e_{22}^{Z Y}\right), \\
\mathcal{V}_{3}(X, T)=k\left(e_{21}^{X T}+e_{32}^{X T}\right), \quad \mathcal{V}_{3}(T, Y)=k\left(e_{12}^{T Y}+e_{23}^{T Y}\right), \\
\mathcal{V}_{3}(Z, X)=\mathcal{V}_{3}(Y, Z)=\mathcal{V}_{3}(T, X)=\mathcal{V}_{3}(Y, T)=\mathcal{V}_{3}(Y, X)=0 .
\end{gathered}
$$




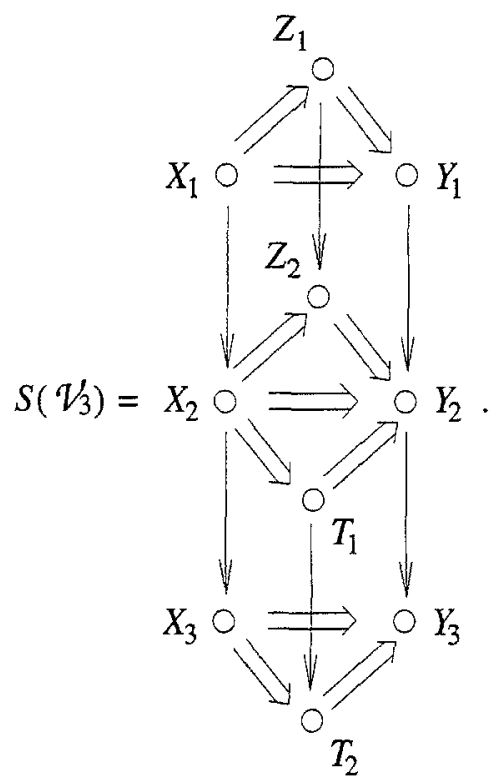

The edges $X_{1} \Rightarrow Y_{1}$ and $X_{3} \Rightarrow Y_{3}$ in the biordered set $S\left(\mathcal{V}_{3}\right)$ are long, but they form a short pair.

Example 2. Consider the vectroid $\mathcal{W}$ with one object $X=\bigoplus_{i=1}^{4} k m_{i}^{X}$ and the following space of morphisms:

$$
\mathcal{W}(X, X)=k 1_{X} \oplus k\left(e_{12}^{X X}+e_{34}^{X X}\right) \oplus k e_{23}^{X X} \oplus\left(\underset{j>i+1}{\oplus} k e_{i j}^{X X}\right)
$$

Then $\operatorname{dim} \mathcal{W}=4$, $\operatorname{def} \mathcal{W}=2$, and

$$
S(\mathcal{W})=\underset{X_{1}}{0} \Rightarrow \underset{X_{2}}{0} \longrightarrow \underset{X_{3}}{0} \Rightarrow \begin{aligned}
& 0 \\
& X_{4}
\end{aligned}
$$

Let $S$ be a locally finite completed biordered set. We define the relations $\leq, \triangleleft$, and $\approx$ on the set $S \times \mathbb{N}$ in the following way:

(i) $(s, i) \leq(t, j)$ if $s \leq t$;

(ii) $(s, i) \triangleleft(t, j)$ if $s \triangleleft t$;

(iii) $(s, i) \approx(t, j)$ if $s \approx t$ and $i=j$.

Note that $\leq$ is a quasiorder ([13], II.1), $\triangleleft$ defines an ideal in the category Cat $(S \times \mathbb{N}, \leq)$ associated with the quasiordered set $(S \times \mathbb{N}, \leq)$, and $\approx$ is the equivalence relation on $S \times \mathbb{N}$.

A function $\varphi: S \rightarrow \mathbb{N}_{0}\left(\mathbb{N}_{0}=\mathbb{N} \cup 0\right)$ is called the dimension of $\varphi$ on the set $S$ if $\varphi(s)=\varphi(t)$ for $s \approx t$. Denote $S_{\varphi}=\{(s, i) \in S \times \mathbb{N} \mid i \leq \varphi(s)\}$.

A matrix $M$ (which may have no columns or rows) is called a representation of $S$ of dimension $\varphi$ if its columns $M_{i}$ are enumerated by the elements of the set $S_{\varphi}$, i.e., the bijection $n:\{1, \ldots, l\} \rightarrow S_{\varphi}$ is given, where $l$ is the number of columns of $M$. The columns $M_{i}$ and $M_{j}$ of the representation $M$ are called comparable (equivalent) if $n(i)$ and $n(j)$ are comparable with respect to $\leq$ (are equivalent with respect to $\approx$ ) in $S \times \mathbb{N}$. 
The representation $M$ of dimension $\varphi$ is called faithful if $\varphi(s) \neq 0$ for every $s \in S$ and faithful at a point $s \in S$ if $\varphi(s) \neq 0$.

Let $\mathcal{V}$ be a chain vectroid and let $(U, f, X) \in \operatorname{Rep} \mathcal{V}$. Then the choice of a triangular basis of $\mathcal{V}$ and a basis of the space $U$ associates $(U, f, X)$ with a representation of the completed biordered set $S(V)$. However, generally speaking, neither the vectroid $\mathcal{V}$ nor the category $\operatorname{Rep} \mathcal{V}$ is determined by $S(\mathcal{V})$. Thus, in Examples 1 (a) and $1(\mathrm{~b})$, we have $S\left(\mathcal{V}_{1}\right) \simeq S\left(\mathcal{V}_{2}\right)$ while $\mathcal{V}_{1} \neq \mathcal{V}_{2}$; moreover, $\mathcal{V}_{1}$ is finitely represented while $\mathcal{V}_{2}$ is not finitely represented. However, if $\operatorname{def}(\mathcal{V}) \leq 1$, then the category $\operatorname{Rep} \mathcal{V}$ (but not $\mathcal{V}$ itself!) is determined by the completed biordered set $S(\mathcal{V})$ in all known cases. In Sec. 2, we show that a finitely represented vectroid can be uniquely restored on the basis of $S(\mathcal{V})$.

\section{Vectroids of Defect $\leq 1$}

Lemma 1. Let $\mathcal{V}$ be a chain vectroid and let $X \in \mathcal{V}$. Then

(a) all nonzero submodules of the module $X_{\mathcal{V}(X X)}$ are cyclic, i.e.,

$$
X=X_{1} \underset{\ddagger}{\supset} X_{2} \underset{\ddagger}{\supset} \underset{\ddagger}{\supset} X_{d(X)} \underset{\ddagger}{\supsetneq} 0, \quad X_{i}=m_{i}^{X} \mathcal{V}(X, X) ;
$$

(b) the generators $m_{1}^{X}, \ldots, m_{d(X)}^{X}$ form a $k$-basis of the space $X$;

(c) they can be chosen so that, for some $\varphi_{X} \in \operatorname{Rad}_{\mathcal{V}}(X, X)$,

$$
m_{2}^{X}=m_{1}^{X} \varphi_{X}, \ldots, \quad m_{d(X)}^{X}=m_{1}^{X} \varphi_{X}^{d(X)-1}
$$

and $\varphi_{X}^{d}=0$.

\section{Proof.}

(a) If $X_{1}$ is not cyclic, then it can be represented as the sum of two submodules $N_{1}+N_{2}$ such that $N_{1} D N_{2}$ and $N_{1} \nsubseteq N_{2}$.

(b) Since the field $k$ is algebraically closed and the algebra $\mathcal{V}(X, X)$ is local, the simple module $X_{i} / X_{i+1}$ $(1 \leq i<d(X))$ is isomorphic to $k$.

(c) We can take $m_{1}^{X} \in X_{1} \backslash X_{2}$ and $\varphi_{X} \in \operatorname{Rad}_{\mathcal{V}}(X, X) \backslash\left(P_{1} \cup \ldots \cup P_{d(X)-1}\right)$, where $P_{i}=\{\psi \in \mathcal{V}(X, X) \mid$ $\left.X_{i} \Psi \subset X_{i+2}\right\}$ are proper subspaces of the space $\operatorname{Rad}_{\mathcal{V}}(X, X)$ and $X_{d(X)+1}=0$.

Denote by $\mathrm{r}(\mathcal{V})$ the least possible rank of a basis of a vectroid $\mathcal{V}$.

Lemma 2. Let $\mathcal{V}$ be a chain vectroid. Then

(a) $\operatorname{def}(\mathcal{V}) \geq 0$; furthermore, $\operatorname{def}(\mathcal{V})=0$ if and only if $\mathrm{r}(\mathcal{V}) \leq 1$;

(b) $\operatorname{def}(\mathcal{V}) \geq \mathrm{r}(\mathcal{V})-1$ 
Proof. Let $X, Y \in \mathcal{V}$. We choose bases $m_{1}^{X}, \ldots, m_{d(X)}^{X}$ and $m_{1}^{Y}, \ldots, m_{d(X)}^{Y}$ in $X$ and $Y$ as in Lemma 1. Since $\operatorname{Rad}_{\mathcal{V}}(X, Y)$ is closed with respect to the multiplication by $\varphi_{X}$ from the left and by $\varphi_{Y}$ from the right, the space of $d(X) \times d(Y)$ matrices

$$
R(X, Y)=\left\{\left(\alpha_{i j}\right) \mid \sum \alpha_{i j} e_{i j}^{X Y} \in \operatorname{Rad}_{\mathcal{V}}(X, X)\right\}
$$

[which defines $\left.\operatorname{Rad}_{\mathcal{V}}(X, Y)\right]$ is stable under shifts rightward and upward. Therefore, it has the following staircase form (cf. [1], 4.7):

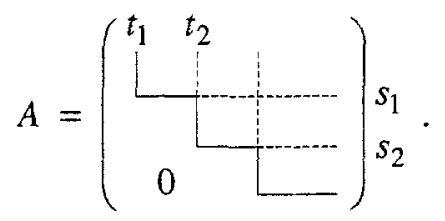

I. Let us prove that $\left|\left\{\left(X_{i}, Y_{j}\right) \mid X_{i}<Y_{j}\right\}\right|$ is equal to the number of elements of $A$ from $R(X, Y)$ located on or above the stairs.

Indeed, the set of nodes

$$
\begin{gathered}
\mathcal{K}_{X Y}=\left\{\left(s_{1}, t_{1}\right), \ldots,\left(s_{n}, t_{n}\right)\right\} \\
\left(1 \leq s_{1}<\ldots<s_{n} \leq d(X), \quad 1 \leq t_{1}<\ldots<t_{n} \leq d(Y)\right)
\end{gathered}
$$

consists of all minimal elements of the set

$$
N_{X Y}=\left\{(s, t) \mid \exists\left(\alpha_{i j}\right) \in R(X, Y): \alpha_{s t} \neq 0\right\}
$$

with respect to the following partial-ordering relation: $(i, j) \leq\left(i^{\prime}, j^{\prime}\right)$ if $i \geq i^{\prime}$ and $j \leq j^{\prime}$. It is clear that $N_{X Y}$ coincides with the set of indexing pairs of elements of the matrix $A$ located on or above the stairs, i.e.,

$$
N_{X Y}=\left\{(i, j) \mid \exists l: 1 \leq i \leq s_{l}, t_{l} \leq j \leq d(Y)\right\} .
$$

Let $(l, r) \in N_{X Y}$. We select $\varphi=\sum \alpha_{i j} e_{i j}^{X Y} \in \operatorname{Rad}(X, Y)$ such that $\alpha_{l r} \neq 0$ and choose $f \in k[x]$ such that $\varphi f\left(\varphi_{Y}\right)=\sum \beta_{i j} e_{i j}^{X Y}$, where $\beta_{l j}=0$ for $j \neq r$, and $\beta_{l r} \neq 1$. Then $m_{l}^{X} \varphi f\left(\varphi_{Y}\right)=m_{r}^{Y}, X_{l}<X_{r}$. Hence, there exists a bijection $N_{X Y} \rightarrow\left\{\left(X_{i}, Y_{j}\right) \mid X_{i}<Y_{j}\right\}$ and $\operatorname{def}(X, Y)=\left|N_{X Y}\right|-\operatorname{dim} \operatorname{Rad}(X, Y)$.

II. Let $A_{\alpha}=\left(a_{i j}^{(\alpha)}\right), 1 \leq \alpha \leq t$, be a $k$-basis of the matrix space $R(X, Y)$. Then there exists a collection of indices $J=\left\{\left(i_{1}, j_{1}\right), \ldots,\left(i_{t}, j_{t}\right)\right\}$ such that the matrices $B_{\alpha}=\left(b_{i j}^{(\alpha)}\right), 1 \leq \alpha \leq t$, are linearly independent. Here, $b_{i j}^{(\alpha)}=a_{i j}^{(\alpha)}$ for $(i, j) \in J$, and $b_{i j}^{(\alpha)}=0$ for $(i, j) \notin J$. By using transformations of the form $A_{\alpha} \rightarrow A_{\alpha}+$ $a A_{\beta}(\alpha \neq \beta, a \in k)$, we can pass from $A_{1}, \ldots, A_{t}$ to a new $k$-basis $C_{\alpha}=\left(c_{i j}^{(\alpha)}\right), 1 \leq \alpha \leq t$, such that $c_{i_{\beta} j_{\beta}}^{(\alpha)}=0$ for $\alpha \neq \beta$ and $c_{i_{\alpha} j_{\alpha}}^{(\alpha)} \neq 0$. Since every matrix $C_{\alpha}$ has the same staircase form as $A$, and $C_{\alpha}$ has at most $t-1$ zero elements among those located above the stairs, we conclude that $C_{\alpha}$ has at most $\left|N_{X Y}\right|-t+1$ nonzero elements. Therefore, $\operatorname{rank}\left(C_{\alpha}\right) \leq\left|N_{X Y}\right|-t+1, \mathrm{r}(X, Y) \leq\left|N_{X Y}\right|-\operatorname{dim} R(X, Y)+1$, and $\operatorname{def}(X, Y) \geq \mathrm{r}(X, Y)-1$, which proves (b). 
III. Let us prove (a). By virtue of (b) and step I of the proof, it is sufficient to show that $r(\mathcal{V})=1$ yields $\operatorname{def}(\mathcal{V})=0$.

Let $A=\left(a_{i j}\right) \in R(X, Y)$ and let $\operatorname{rank}(A)=1$. Since $R(X, Y)$ is stable under shifts rightward and upward, it contains matrix units $E_{i j}$ for all $(i, j) \geq(s, t)$, where $(s, t)$ is the minimal element of the set $\left\{(i, j) \mid \alpha_{i j} \neq 0\right\}$ with respect to the partial-ordering relation defined in step I. Therefore, if $\mathrm{r}(X, Y)=1$, then $R(X, Y)$ has a basis including matrix units and, by virtue of $\operatorname{step} I, \operatorname{def}(X, Y)=0$. Lemma 2 is proved.

Proposition 1. A chain vectroid whose defect does not exceed one has a scalarly multiplicative basis whose rank does not exceed two.

Proof. We use the notation from the proof of Lemma 2.

I. Assume that $X, Y \in \mathcal{V}$. Let us show that there exist $c_{1}^{X Y}, \ldots, c_{n}^{X Y} \in k$ (possibly, all equal to zero) such that

$$
\operatorname{Rad}_{\mathcal{V}}(X, Y)=\left\{\sum_{(i, j) \in N_{X Y}} \alpha_{i j} E_{i j}^{X Y} \mid c_{1}^{X Y} \alpha_{s_{1} t_{1}}+\ldots+c_{n}^{X Y} \alpha_{s_{n} t_{n}}=0\right\}
$$

Indeed, by virtue of the condition $\operatorname{def}(\mathcal{V}) \leq 1$, the codimension of the space $R(X, Y)$ in the space of all staircase matrices of the form $A$ does not exceed one. Therefore, there exist $c_{i j} \in k$ such that

$$
R(X, Y)=\left\{\sum_{(i, j) \in N_{X Y}} \alpha_{i j} E_{i j} \mid \sum_{(i, j) \in N_{X Y}} c_{i j} \alpha_{i j}=0\right\},
$$

where $E_{i j}$ are matrix units.

Let $c_{i r} \neq 0$ for some $(i, r) \notin \mathcal{K}_{X Y}$ Then $R(X, Y)$ contains $B_{i j}=E_{i j}+\gamma_{i j} E_{l r}$ for all $(i, j) \in N_{X Y} \backslash(i, r)$, where $\gamma_{i j}=-c_{i j} c_{l r}^{-1}$. We fix $(s, t) \in \mathcal{K}_{X Y}$ such that $(s, t)<(l, r)$ and set $\left(l_{i}, r_{i}\right)=(s, t)+i[(l, r)-(s, t)]$. Let $m$ be such that $\left(l_{m}, r_{m}\right) \in N_{X Y}$ but $\left(l_{m+1}, r_{m+1}\right) \notin N_{X Y}$. Let $F_{1}$ and $F_{2}$ be the matrices of the maps $\varphi_{X}$ and $\varphi_{Y}$, respectively (see Lemma 1 ). Then $R(X, Y)$ contains the matrices

$$
\begin{gathered}
B_{s t}=E_{l_{0} r_{0}}+\gamma_{s t} E_{l_{1} r_{1}}, \quad F_{1}^{s-l} B_{s t} F_{2}^{r-t}=E_{l_{1} r_{1}}+\gamma_{s t} E_{l_{2} r_{2}}, \\
F_{1}^{2(s-l)} B_{s t} F_{2}^{2(r-t)}=E_{l_{2} r_{2}}+\gamma_{s t} E_{l_{3} r_{3}}, \ldots, E_{l_{m} r_{m}} .
\end{gathered}
$$

Hence, it contains the matrix $E_{l_{1} r_{1}}=E_{l r}$, which contradicts the assumption that $c_{i r} \neq 0$.

Therefore, we have $c_{i j}=0$ for all $(i, j) \in \mathcal{K}_{X Y}$, which was to be proved.

II. A scalarly multiplicative basis of the vectroid $\mathcal{V}$ can be obtained by supplementing the vectors $m_{i}^{X}$ with morphisms $f_{l}^{X Y}$ of the following form:

(a) $e_{i j}^{X Y}$ for $(i, j) \in N_{X Y} \backslash \mathcal{K}_{X Y}$ and for $(i, j)=\left(s_{r}, t_{r}\right) \in \mathcal{K}_{X Y}$ such that $c_{r}^{X Y}=0$;

(b) $\quad e_{s_{r(1)} t_{r(1)}}^{X Y}-\left(c_{r(1)}^{X Y} / c_{r(i)}^{X Y}\right) e_{s_{r(i)} t_{r(i)}}^{X Y}$ for all $2 \leq i \leq q$, where $\{r(1), \ldots, r(q)\}=\left\{r \mid c_{r}^{X Y} \neq 0\right\}$ and $r(1) \leq \ldots \leq r(q)$. 
Lemma 3. Let $\mathcal{V}$ be a vectroid of defect $\leq 1$. The completed biordered set $S(\mathcal{V})$ satisfies the following conditions:

I. The equivalence class of every element is linearly ordered.

II. If $a_{1}<a_{2}<\ldots<a_{n}$ and $a_{1} \Rightarrow a_{n}$, then there exist $a_{1}^{\prime} \sim a_{1}, \ldots, a_{n}^{\prime} \sim a_{n}$ such that $a_{1}^{\prime}<$ $a_{2}^{\prime}<\ldots<a_{n}^{\prime}$ and $a_{1}^{\prime} \Rightarrow a_{n}^{\prime}$.

III. If ed $(a, b) \geq 3$, then the edge $a \Rightarrow b$ is maximal.

IV. $a \triangleleft a$ if and only if $d(a)=1$.

Proof. Let $\mathcal{V}$ be a chain vectroid whose defect does not exceed one and let $\left\{\left(m_{i}^{X}\right),\left(f_{l}^{X Y}\right)\right\}$ be its scalarly multiplicative basis of rank two constructed in the proof of Proposition 1.

Condition I follows from the equality $m_{i}^{X} \varphi_{X}=m_{i+1}^{X}[i<\operatorname{dim}(X)]$.

It is sufficient to verify condition II for $n=2$ and $n=3$. For $n=2$, the proof is obvious. Indeed, let $X_{i} \Rightarrow Y_{j}$. Then $m_{i}^{X} f_{l}^{X Y}=\alpha m_{j}^{Y}$ for some $f_{l}^{X Y}=\alpha e_{i j}^{X Y}+\beta e_{i^{\prime} j^{\prime}}^{X Y}, i \neq i^{\prime}, j \neq j^{\prime}, \alpha, \beta \in k^{*}$, i.e., $X_{i^{\prime}} \Rightarrow X_{j^{\prime}}$. Let us prove condition II for $n=3$. Assume that $X_{i}<Y_{j}<Z_{r}$ and $X_{i} \Rightarrow Z_{r}$. Then there exist $f_{l}^{X Y}$ and $f_{p}^{Y Z}$ such that $m_{i}^{X} f_{l}^{X Y}=\alpha m_{j}^{Y}$ and $m_{j}^{Y} f_{p}^{Y Z}=\beta m_{r}^{Z}, \alpha \neq 0 \neq \beta$. Since $X_{i} \Rightarrow Z_{r}$, we have rank $\left(f_{l}^{X Y} f_{p}^{Y Z}\right) \neq 1$. Consequently, $f_{l}^{X Y}=\alpha e_{i j}^{X Y}+\gamma e_{i^{\prime} j^{\prime}}^{X Y}, \quad f_{p}^{Y Z}=\beta e_{j r}^{Y Z}+\delta e_{j^{\prime} r^{\prime}}^{Y Z}, \quad \gamma \neq 0 \neq \delta$, and $X_{i^{\prime}}<Y_{j^{\prime}}<Z_{r^{\prime}}$. If $X_{i^{\prime}} \varangle Z_{r^{\prime}}$, then $e_{i^{\prime} r^{\prime}}^{X Z} \in$ $\operatorname{Rad}(X, Z)$, and $f_{l}^{X Y} f_{p}^{Y Z}-\gamma \delta e_{i^{\prime} r^{\prime}}^{X Z}$ has rank one and maps $m_{i}^{X}$ onto $\alpha \beta m_{r}^{Z}$, which contradicts the assumption that $X_{i} \Rightarrow Z_{r^{\prime}}$. Therefore, $X_{i^{\prime}} \Rightarrow Z_{r^{\prime}}$.

Let us prove condition III. Let $X_{i} \Rightarrow Y_{j}, X_{i^{\prime}} \Rightarrow Y_{j^{\prime}}$, and $X_{i^{\prime \prime}} \Rightarrow Y_{j^{\prime \prime}}$ be different edges. Assume, e.g., that $X_{i} \Rightarrow Y_{j}$ is not maximal from the right, i.e., there exists $Z_{r}>Y_{j}$ such that $X_{i} \Rightarrow Z_{r}$. Then $X_{i} \Rightarrow Y_{j} \Rightarrow Z_{r}$ and there is a basic morphism $f_{l}^{Y Z}=\alpha e_{j r}^{Y Z}+\beta e_{t r^{\prime}}^{Y Z}, \alpha \neq 0 \neq \beta, j \neq t$. We may take $j^{\prime} \neq t$. According to step I of the proof of Proposition 1, there exists $\psi=\gamma e_{i j}^{X Y}+\delta e_{i^{\prime} j^{\prime}}^{X Y} \in \operatorname{Rad}_{\mathcal{V}}(X, Y), \gamma \neq 0 \neq \delta$. The existence of the morphism $\psi f_{l}^{Y Z}=\alpha \gamma e_{i r}^{X Z}$ contradicts $X_{i} \Rightarrow Z_{r}$.

Condition IV follows from the fact that the ring $\mathcal{V}(X, X)$ is local: If $m_{i}^{X} f=m_{i}^{X}$, then $f=1$ and $\operatorname{rank}(f)=$ $\operatorname{dim} X$.

Lemma 4. The following property is a consequence of conditions I-III: If $(a \Rightarrow b)-\left(a^{\prime} \Rightarrow b^{\prime}\right)$, then either $a<a^{\prime}$ and $b<b^{\prime}$ or $a>a^{\prime}$ and $b>b^{\prime}$.

Proof. Let $a=a^{\prime}$. By condition II, there exists an edge $\left(a^{\prime \prime} \Rightarrow b^{\prime \prime}\right) \sim(a \Rightarrow b)$ such that $a^{\prime \prime} \neq a$ and $b^{\prime \prime} \neq b$. Hence, ed $(a, b) \geq 3$ and the edges $a \Rightarrow b$ and $a \Rightarrow b^{\prime}$ are maximal by virtue of condition III. However, $b \approx b^{\prime}, a \Rightarrow b^{\prime}$. By condition I, $b<b^{\prime}$ or $b>b^{\prime}$, and $a \Rightarrow b$ or $a \Rightarrow b^{\prime}$ is not maximal. Therefore, $a \neq a^{\prime}$ and, similarly, $b \neq b^{\prime}$. If, for example, $a<a^{\prime}$ and $b>b^{\prime}$, then, since $a<a^{\prime}<b^{\prime}<b$ and $a \Rightarrow b$, we get $a \Rightarrow b^{\prime}$. However, we have just proved that such an edge does not exist. Therefore, we have either $a<a^{\prime}$ and $b<b^{\prime}$ or $a>a^{\prime}$ and $b>b^{\prime}$.

\section{Lemma 5.}

(a) Let $\operatorname{char}(k) \neq 2$ and let $\mathcal{V}$ be a chain vectroid whose defect does not exceed one. If $\mathcal{V}$ has a multiplicative basis of rank at most two, then $S(\mathcal{V})$ satisfies the following condition: 
V. If $\left(\alpha_{1}, \alpha_{2}\right),\left(\alpha_{2}, \alpha_{3}\right), \ldots,\left(\alpha_{t}, \alpha_{t+1}\right)$ are long pairs of edges and $t$ is even, then $\left(\alpha_{1}, \alpha_{t+1}\right)$ is a short pair of edges.

(b) On the basis of a locally finite completed biordered set $S$ satisfying conditions $I-V$, one can construct a chain vectroid $\mathcal{V}$ with defect $\leq 1$ that has a multiplicative basis and for which $S(\mathcal{V}) \simeq S$.

Proof. (a) Assume that $\mathcal{V}$ is a chain vectroid whose defect does not exceed one and $S(\mathcal{V})$ contains long pairs of edges $\left(\alpha_{1}, \alpha_{2}\right),\left(\alpha_{2}, \alpha_{3}\right), \ldots,\left(\alpha_{t}, \alpha_{t+1}\right),\left(\alpha_{1}, \alpha_{t+1}\right)$, where $\alpha_{l}: X_{i} \Rightarrow Y_{j_{l}}(1 \leq l \leq t+1)$. Since $\left(\alpha_{1}, \alpha_{2}\right)$ is a long pair, we have $X_{i_{l}} \Rightarrow Z_{p_{l}} \Rightarrow Y_{j_{l}}(l=1,2)$ for some $Z$. According to condition III, ed $(X, Z)=$ ed $(Z, Y)=2$. Assume that $\mathcal{V}$ has a multiplicative basis whose rank does not exceed two. Then $e_{i_{1} p_{1}}^{X Z}+e_{i_{2} p_{2}}^{X Z}$ and $e_{p_{1} j_{1}}^{Z Y}+e_{p_{2} j_{2}}^{Z Y}$ are basic morphisms and $e_{1}+e_{2} \in \mathcal{V}(X, Y)$, where $e_{l}=e_{i_{l} j_{l}}^{X Y}(1 \leq l \leq t+1)$. By analogy, $e_{2}+e_{3}, \ldots, e_{t}+e_{t+1}, e_{1}+e_{t+1} \in \mathcal{V}(X, Y)$. For even $t$ and $\operatorname{char}(k) \neq 2$, we get $e_{1}=e_{i_{1} j_{1}}^{X Y} \in \mathcal{V}(X, Y)$, which contradicts $X_{i_{1}} \Rightarrow X_{j_{1}}$.

(b) Assume that $S$ is a locally finite completed biordered set satisfying conditions I-V. Let us construct a vectroid $\mathcal{V}=\operatorname{Vect}(S)$ whose objects are the vector spaces $X=k x_{1} \oplus \ldots \oplus k x_{d X}$, where $\left\{x_{1}, \ldots, x_{d(X)}\right\} \subset S$ are equivalence classes and $x_{1}<x_{2}<\ldots<x_{d(X)}$. The space $\operatorname{Rad}_{\mathcal{V}}(X, Y)$ is generated by linear maps $e_{i j}^{X Y}$ for all $x_{i} \triangleleft y_{j}$ and by all maps from a certain set $L(X, Y) \subset\left\{e_{i j}^{X Y}+e_{i^{\prime} j^{\prime}}^{X Y} \mid\left(x_{i} \Rightarrow y_{j}\right) \sim\left(x_{i^{\prime}} \Rightarrow y_{j^{\prime}}\right)\right\}$. This set contains $e_{i j}^{X Y}+e_{i^{\prime} j^{\prime}}^{X Y}$ for all long pairs $\left(x_{i} \Rightarrow y_{j}, x_{i^{\prime}} \Rightarrow y_{j^{\prime}}\right)$ and is maximal with respect to the following property: The linear span of $L(X, Y)$ contains no maps of the form $e_{i j}^{X Y}$. In order to uniquely choose $L(X, Y)$, we impose the following condition: If $\left(x_{i} \Rightarrow y_{j}, x_{i^{\prime}} \Rightarrow y_{j^{\prime}}\right)$ and $\left(x_{s} \Rightarrow y_{t}, x_{s^{\prime}} \Rightarrow y_{t^{\prime}}\right), i<i^{\prime}, s<s^{\prime}$, are two short pairs and $e_{i j}^{X Y}+e_{i^{\prime} j^{\prime}}^{X Y} \in L(X, X), e_{s t}^{X Y}+e_{s^{\prime} t^{\prime}}^{X Y} \notin L(X, X)$, then either $i<s$ or $i=s, i^{\prime}<s^{\prime}$. Note that we construct a vectroid with fixed multiplicative basis.

Let us prove that $\mathcal{V}$ is well defined, i.e., that $f g \in \operatorname{Rad}_{\mathcal{V}}(X, Y)$ for all $f \in \operatorname{Rad}_{\mathcal{V}}(X, Y)$ and $g \in \operatorname{Rad}_{\mathcal{V}}(Y, Z)$. We can assume that $f$ and $g$ are generating maps [i.e., $f=e_{i j}^{X Y}, x_{i} \triangleleft y_{j}$, or $f \in L(X, Y)$ ]. Let $f=e_{i j}^{X Y}+\ldots$, $g=e_{j l}^{Y Z}+\ldots$ (hence, $\left.x_{i}<y_{j}<z_{l}\right)$. If $x_{i} \triangleleft z_{l}$, then $e_{i l}^{X Z} \in \operatorname{Rad}_{\mathcal{V}}(X, Z)$ and $f g \in \operatorname{Rad}_{\mathcal{V}}(X, Z)$. Now assume that $x_{i} \Rightarrow z_{l}$. Then $x_{i} \Rightarrow y_{j} \Rightarrow z_{l}$ and, according to condition $\Pi$, there exist other $x_{i^{\prime}} \Rightarrow z_{l^{\prime}}, x_{i^{\prime}} \Rightarrow y_{j^{\prime}} \Rightarrow z_{l^{\prime}}$. By virtue of condition III, ed $\left(x_{i}, y_{j}\right)=\mathrm{ed}\left(y_{j}, z_{l}\right)=2$. Therefore, $f=e_{i j}^{X Y}+e_{i^{\prime} j^{\prime}}^{X Y}$ and $g=e_{j l}^{Y Z}+e_{j^{\prime} l^{\prime}}^{Y Z}$. Since $\left(x_{i} \Rightarrow z_{l}, x_{i^{\prime}} \Rightarrow z_{l^{\prime}}\right)$ is a long pair of edges, we get $f g=e_{i l}^{X Z}+e_{i^{\prime} l^{\prime}}^{X Z} \in \operatorname{Rad}_{\mathcal{V}}(X, Z)$ by the definition of $L(X, Z)$.

Let us investigate the form of $\operatorname{Rad}_{\mathcal{V}}(X, Y)$. Let $\mathcal{K}_{X Y}=\left\{\left(s_{1}, t_{1}\right), \ldots,\left(s_{n}, t_{n}\right)\right\}$ be the set of minimal elements of the set $N_{X Y}=\left\{(i, j) \mid x_{i}<y_{j}\right\}$. If $(i, j) \in N_{X Y}$ and $(i, j)<\left(i^{\prime}, j^{\prime}\right)$, then $x_{i} \leq x_{i}<y_{j} \leq y_{j^{\prime}}$. Hence, $\left(i^{\prime}, j^{\prime}\right) \in N_{X Y}$ and $N_{X Y}$ has the form described in step I of the proof of Lemma 2. By Lemma 4, if $x_{i} \Rightarrow y_{j}$, then $x_{i}, \triangleleft y_{j^{\prime}}$ and $(i, j) \in \mathcal{K}_{X Y}$. Since $L(X, Y)$ is maximal, the space $\operatorname{Rad}_{\mathcal{V}}(X, Y)$ has the form described in step I of the proof of Proposition 1 , and $\operatorname{def}(X, Y) \leq 1$. Lemma 5 is proved.

The category $\operatorname{Rep}(\mathcal{V})$ of representations of the vectroid $\mathcal{V}=\operatorname{Vect}(S)$ constructed in the proof of assertion (b) of Lemma 5 is called the category $\operatorname{Rep}(S)$ of representations of a locally finite completed biordered set $S$ satisfying conditions I-V.

Remark 4. If $\mathcal{V}$ is a chain vectroid such that $\mathrm{r}(\mathcal{V})=2$ and $\operatorname{def}(\mathcal{V})>1$, we can define an equivalence relation $\approx_{\text {ed }}$ on the set of edges of $S(\mathcal{V})$. For this purpose, we set $\left(X_{i} \Rightarrow Y_{j}\right) \approx_{\text {ed }}\left(X_{i^{\prime}} \Rightarrow Y_{j^{\prime}}\right)$ if and only if there exists a morphism $\xi \in \mathcal{V}(X, Y)$ such that 


$$
\begin{gathered}
X_{i} \xi \subset Y_{j}, \quad\left(X_{i} \operatorname{Rad}_{\mathcal{V}(X, X)}\right) \xi \subset Y_{j} \operatorname{Rad}_{\mathcal{V}(Y, Y)}, \\
X_{i^{\prime}} \xi \subset Y_{j^{\prime}}, \quad\left(X_{i^{\prime}} \operatorname{Rad}_{\mathcal{V}(X, X)}\right) \xi \subset Y_{j^{\prime}} \operatorname{Rad}_{\mathcal{V}(Y, Y)}, \\
X_{i} \xi \nsubseteq Y_{j} \operatorname{Rad}_{\mathcal{V}(Y, Y)}, \quad X_{i^{\prime}} \xi \nsubseteq Y_{j^{\prime}} \operatorname{Rad}_{\mathcal{V}(Y Y)},
\end{gathered}
$$

and there is no $\zeta \in \mathcal{V}(X, Y)$ such that $m_{i}^{X} \zeta=m_{j}^{X}, m_{i^{\prime}}^{X} \zeta=0$. On the basis of the completed biordered set $S$ with given equivalence relation $\approx$ ed on edges, we can similarly define a vectroid $\operatorname{Vect}(S, \approx$ ed $)$ such that $S\left(\operatorname{Vect}\left(S, \approx_{\text {ed }}\right)\right) \approx\left(S, \approx_{\text {ed }}\right)$. Note that if $\operatorname{def} \mathcal{V} \leq 1$, then the equivalence relation $\approx_{\text {ed }}$ coincides with the equivalence relation for edges introduced in Sec. 1 .

Lemma 6. If a vectroid $\mathcal{V}$ is finitely represented, then $\operatorname{def} \mathcal{V} \leq 1$ and the completed biordered set $S(\mathcal{V})$ satisfies conditions $I-V$ and the following one:

VI. $d(a) \leq 3$ for all $a \in S(\mathcal{V})$.

Proof. Condition VI is satisfied because $\mathcal{V}$ is a chain vectroid and $\operatorname{dim} \mathcal{V} \leq 3$. Let us prove that def $\mathcal{V} \leq 1$. According to Lemma 1 in [4], if $\operatorname{dim} X=2$, then

(i) $\mathcal{V}(X, X)=k 1_{X} \oplus k e_{12}^{X X}$,

and if $\operatorname{dim} X=3$, then

(ii) $\mathcal{V}(X, X)=k 1_{X} \oplus k e_{12}^{X X} \oplus k e_{23}^{X X} \oplus k e_{13}^{X X}$

or

(iii) $\mathcal{V}(X, X)=k 1_{X} \oplus k\left(e_{12}^{X X}+\lambda e_{23}^{X X}\right) \oplus k e_{13}^{X X}, \lambda \in k^{*}$.

It is clear that $\operatorname{def}(X, X)=0$ in cases (i) and (ii) and $\operatorname{def}(X, X)=1$ in case (iii).

Let $X, Y \in \mathcal{V}$ and let $X \neq Y$. According to Lemma 5 in [4] (this also follows from the proof of Lemma 2), the space $\mathcal{V}(X, Y)$ admits a basis consisting of linear maps of the form $e_{i j}^{X Y}$ and $e_{i j}^{X Y}+\alpha e_{r l}^{X Y}(\alpha \neq 0, i \neq r$, and $j \neq l$ ). Moreover, it contains at most two morphisms of the second form, and if there are two such morphisms, then they have the form $e_{11}^{X Y}+\lambda e_{22}^{X Y}, e_{11}^{X Y}+\mu e_{33}^{X Y}, \lambda, \mu \in k^{*}$. Therefore, $\operatorname{def}(X, Y)=1$ and $\operatorname{def} \mathcal{V} \leq 1$. By virtue of Lemma 2, $S(\mathcal{V})$ satisfies conditions I-IV. Condition V is satisfied because the equivalence class containing more than two edges has the following form: $\left\{x_{i} \Rightarrow y_{j}: i=1,2,3\right\}$. According to Proposition 1 in [4], it contains a short pair of edges.

Proposition 2. The map $\mathcal{V} \mapsto S(\mathcal{V})$ establishes a bijection between the isoclasses of finitely represented vectroids and the isoclasses of finitely represented locally finite completed biordered sets satisfying conditions I-VI.

Proof. Let $M_{1}$ be the class of all finitely represented vectroids and let $M_{2}$ be the class of all finitely represented locally finite completed biordered sets satisfying conditions I-VI. By virtue of Lemma 6 , if $\mathcal{V} \in M_{1}$, then $S(\mathcal{V}) \in M_{2}$. In the proof of Lemma 5 , we have constructed, for every $S \in M_{2}$, a vectroid Vect $(S) \in M_{1}$ such that $S(\operatorname{Vect}(S)) \simeq S$. It remains to prove that $\operatorname{Vect}(S(\mathcal{V})) \simeq \mathcal{V}$ for all $\mathcal{V} \in M_{1}$. 
Let $\mathcal{V} \in M_{1}$. Every space $\operatorname{Rad}_{\mathcal{V}}(X, Y)$ has the form described in step I of the proof of Proposition 1, where $\left(s_{1}, t_{1}\right), \ldots,\left(s_{n}, t_{n}\right)$ are minimal elements of the set $N_{X Y}=\left\{(i, j) \mid X_{i}<Y_{j}, X_{i}, Y_{j} \in S(\mathcal{V})\right\}$. By analogy with the proof of Lemma $5, S(\mathcal{V})$ defines the set of linear maps $L(X, Y)$. According to Propositions 1 and 2 in [4], the bases $\left(m_{1}^{X}, \ldots, m_{d(X)}^{X}\right)$ of the spaces $X \in \mathcal{V}$ can be chosen so that $L(X, Y) \subset \operatorname{Rad}_{\mathcal{V}}(X, Y)$, and $m_{i}^{X}$ together with $e_{i j}^{X Y}\left(X_{i} \triangleleft Y_{j}\right)$ and morphisms from $L(X, Y)$ forms a multiplicative basis of the vectroid $\mathcal{V}$. Thus, we get $\operatorname{Vect}(S(\mathcal{V})) \approx \mathcal{V}$

Remark 5. Two vectroids $\mathcal{V}$ and $\mathcal{V}^{\prime}$ are called locally isomorphic if there exists a bijection $f:$ Ob $\mathcal{V} \rightarrow$ Ob $\mathcal{V}^{\prime}$ and, for every pair of objects $X, Y \in \mathcal{V}$ (including $X=Y$ ), there exist nondegenerate linear maps $\varphi$ : $X \rightarrow f(X)$ and $\psi: Y \rightarrow f(Y)$ such that $\mathcal{V}(X, Y) \psi=\varphi \mathcal{V}^{\gamma}(f(X), f(Y))$. Let us prove the following assertion: If finitely represented vectroids $\mathcal{V}$ and $\mathcal{V}^{\prime}$ are locally isomorphic, then they are isomorphic.

It is sufficient to show that the equality $\mathcal{V}(X, Y) \Psi=\varphi \mathcal{V}^{\prime}(f(X), f(Y))$ implies that $\mathcal{K}_{X Y}=\mathcal{K}_{f(X) f(Y}$ and $\left\{r \mid c_{r}^{X Y} \neq 0\right\}=\left\{r \mid c_{r}^{f(X) f(Y)} \neq 0\right\}$ for all $X, Y \in \mathcal{V}$ (because, in this case, $\left.S(\mathcal{V})\right) \simeq S\left(\mathcal{V}^{\prime}\right)$ and we can use Proposition 2).

For $\operatorname{dim} X=2$, the statement is obvious. Let $\operatorname{dim} X=3$ and let $X=Y$. It follows from the proof of Lemma 6 that $\mathcal{V}(X, X)$ has the form $k 1_{X} \oplus k\left(e_{12}^{X X}+e_{23}^{X X}\right) \oplus k e_{13}^{X X}$ or $k 1_{X} \oplus k e_{12}^{X X} \oplus k e_{23}^{X X} \oplus k e_{13}^{X X}$. Since the map $\alpha \mapsto \varphi^{-1} \alpha \psi$ defines an isomorphism of the spaces $\mathcal{V}(X, X)$ and $\mathcal{V}^{\prime}(f(X), f(X))$, we can conclude that $\mathcal{V}(X, X)$ and $\mathcal{V}^{\prime}(f(X), f(X))$ have the same dimension and, hence, the same form.

Let $X \neq Y$. We can write the following equality in the matrix form: $V \Psi=\Phi V^{\prime}$, where $V=R_{\mathcal{V}}(X, Y)$, $V^{\prime}=R_{V^{\prime}}(f(X), f(Y))$, and $\Psi$ and $\Phi$ are the matrices of the maps $\psi$ and $\varphi$, respectively. Since $V=S V R$ and $V^{\prime}=S^{\prime} V^{\prime} R^{\prime}$ for arbitrary upper triangular matrices $S, R, S^{\prime}$, and $R^{\prime}$ with unit diagonal, we can replace the matrices $\Psi$ and $\Phi$ by $R \Psi R^{\prime-1}$ and $S^{-1} \Phi S$ with exactly one nonzero element in each column and each row (i.e., by permutation matrices). However, it is impossible to pass from any staircase form of $R_{\mathcal{V}}(X, Y)$ to another one by permutations. Remark 5 is proved.

\section{3. $S$-Graphs}

Let $S$ denote a completed biordered set. A collection $\left(\mathcal{B}, \Gamma,-, \sim, \varphi_{\mathcal{B}}\right)$ is called an $S$-graph if the following conditions are satisfied:

(i) $\mathcal{B}$ is a finite set (of vertices of the $S$-graph);

(ii) $\Gamma \subset \mathcal{B}$ is a subset (of nondegenerate vertices);

(iii) is a pseudoequivalence relation on $\mathcal{B}$;

(iv) - is a symmetric binary relation on $\Gamma$;

(v) $\varphi_{\mathcal{B}}: \mathcal{B} \rightarrow S$,

and, moreover,

(vi) if $x-y$ belongs to $\Gamma$, then $\varphi_{\mathcal{B}}(x) \preccurlyeq \varphi_{\mathcal{B}}(y) ;\left|\Gamma^{-}(x)\right| \leq 1$ for any $X \in \Gamma$;

(vii) if $x_{1} \sim x_{2}\left(\right.$ in $\mathcal{B}$ ), then $\varphi_{\mathcal{B}}\left(x_{1}\right) \sim \varphi_{\mathcal{B}}\left(x_{2}\right)$ (in $S$ ); $\varphi_{\mathcal{B}}\left(\mathcal{B}^{\approx}(x)\right)=S^{\approx}\left(\varphi_{\mathcal{B}}(x)\right.$ ) for any $x \in \mathcal{B}$. 
The set of vertices of the $S$-graph $\left(\mathcal{B}, \Gamma,-, \sim, \varphi_{\mathcal{B}}\right)$ can be decomposed into nonintersecting subsets $\mathcal{B}^{\approx}(x)$, $x \in \mathcal{B}$. We call them nodes of the $S$-graph. The node $\mathcal{B}^{\approx}(x)$ is called the node of the vertex $x$ or the node that belongs to the equivalence class $S^{\approx}\left(\varphi_{\mathcal{B}}(x)\right)$.

The nodes of the $S$-graph $\mathcal{B}$ form a graph $K(\mathcal{B})$ (nonoriented and, possibly, possessing loops and multiple edges), in which the edges between two nodes $X, Y \in K(\mathcal{B})$ are the pairs $\{x, y\}$, where $x \in X, y \in Y$, and $x-y$.

The $S$-graph $\mathcal{B}$ is called connected if the graph $K(\mathcal{B})$ is connected. The $S$-graph $\mathcal{B}$ is called nondegenerate if $\Gamma \cap \mathcal{B}^{\approx}(x) \neq \varnothing$ for all $x \in \mathcal{B}$.

Morphisms of $S$-graphs are defined in a natural way. In particular, one can speak about isomorphic $S$-graphs and $S$-subgraphs. Denote by $\hat{C}(S)$ the set of isoclasses of connected $S$-graphs.

Remark 6. The definition of an $S$-graph immediately implies that $\left.\varphi_{\mathcal{B}}\right|_{\mathcal{B} \approx(x)}$ is a bijection of $\mathcal{B}^{\approx}(x)$ onto $S \approx\left(\varphi_{\mathcal{B}}(x)\right)$ for every $x \in \mathcal{B}$. A connected $S$-graph is always nondegenerate except the case where $\Gamma=\varnothing$ and $|\mathcal{B} / \approx|=1$.

Remark 7. We depict nondegenerate vertices of the $S$-graph by dots with the values of the map $\varphi_{\mathcal{B}}$ written above them. Two dots corresponding to vertices $x, y \in \Gamma$ are joined by a wavy line if $x-y$ and by a straight line if $x-y$. It follows from Remark 6 that a nondegenerate $S$-graph can be uniquely reconstructed by the quadruple $\left(\Gamma,-, \sim_{\Gamma},\left.\varphi_{\mathcal{B}}\right|_{\Gamma}\right)$, where $\sim_{\Gamma}$ is the restriction of $\sim$ to $\Gamma$.

Remark 8. The definition of an $S$-graph does not take into account the relation $\triangleleft$ on $S$, i.e., it is defined by $S^{w}$. In Sec.5, we define the set $C(S)$ of (connected) $S$-graphs with marked vertex and introduce an order relation depending on $\triangleleft$ on this set.

The sequence of vertices of an $S$-graph $\left(x_{0}^{\prime}, x_{1}, x_{1}^{\prime}, \ldots, x_{n-1}, x_{n-1}^{\prime}, x_{n}\right), n \in \mathbb{N}$, such that $\mathcal{B}^{\approx}\left(x_{0}^{\prime}\right)=X$, $\mathcal{B}^{\approx}\left(x_{n}\right)=Y, x_{i} \sim x_{i}^{\prime}$ if $i=\overline{1, n-1}$, and $x_{i}^{\prime}-x_{i+1}$ if $i=\overline{0, n-1}$, is called a path in the $S$-graph $\mathcal{B}$ from the node $X$ to the node $Y$. Note that $\left(\mathcal{B}^{\approx}\left(x_{0}^{\prime}\right), \mathcal{B}^{\approx}\left(x_{1}\right), \mathcal{B}^{\approx}\left(x_{2}\right), \ldots, \mathcal{B}^{\approx}\left(x_{n}\right)\right)$ is a path in $K(\mathcal{B})$ from $X$ to $Y$. Conversely, if $\left(X=X_{0}, X_{1}, \ldots, X_{n-1}, X_{n}=Y\right)$ is a path in $K(\mathcal{B})$ from $X$ to $Y$, then, in the $S$-graph $\mathcal{B}$, there exists a path $\left(x_{0}^{\prime}, x_{1}, x_{1}^{\prime}, \ldots, x_{n-1}, x_{n-1}^{\prime}, x_{n}\right)$ from $X$ to $Y$ such that $\mathcal{B}^{\approx}\left(x_{i}\right)=X_{i}, i=\overline{1, n}$. "By virtue of Remark 6, a path in the $S$-graph $\mathcal{B}$ consists of only nondegenerate vertices.

An $S$-graph $\mathcal{B}$ is called acyclic if the graph $K(\mathcal{B})$ is a forest (i.e., contains no cycles). A completed biordered set $S$ is called acyclic if every $S$-graph is acyclic. It obviously follows from the definitions introduced that if $\mathcal{B}$ is an acyclic $S$-graph, then

(i) $\mathcal{B}$ is a connected graph if and only if $K(\mathcal{B})$ is a tree,

(ii) if $\mathcal{B}$ is a connected graph, then the path in $\mathcal{B}$ from $X$ to $Y$ exists and is unique for any nodes $X, Y \in$ $K(\mathcal{B})$.

Lemma 7. Let $S$ be acyclic, let $X, Y, Z, T \in K(\mathcal{B})$ be nodes of the connected $S$-graph $\mathcal{B}$ such that $\varphi_{\mathcal{B}}(X) \approx \varphi_{\mathcal{B}}(Y) \approx \varphi_{\mathcal{B}}(Z) \approx \varphi_{\mathcal{B}}(T)$ in $S$, let $\left(x_{0}^{\prime}, \ldots, x_{n}\right)$ be a path in $\mathcal{B}$ from $X$ to $Y$, and let $\left(z_{0}^{\prime}, \ldots, z_{m}\right)$ be a path in $\mathcal{B}$ from $Z$ to $T$. Then the following relations hold:

(a) $\varphi_{\mathcal{B}}\left(x_{0}^{\prime}\right)=\varphi_{\mathcal{B}}\left(x_{n}\right)$, 
(b) $\varphi_{\mathcal{B}}\left(z_{0}^{\prime}\right)=\varphi_{\mathcal{B}}\left(x_{0}^{\prime}\right)$.

Proof. (a) Let $\varphi_{\mathcal{B}}\left(x_{0}^{\prime}\right) \neq \varphi_{\mathcal{B}}\left(x_{n}\right)$. Then

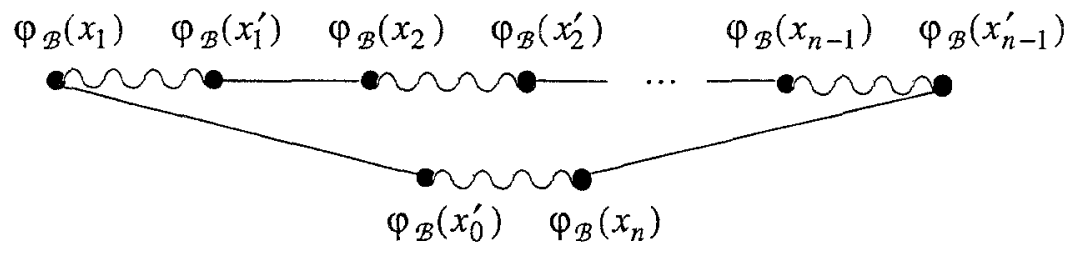

is an $S$-graph, which contradicts the assumption that $S$ is acyclic.

(b) By virtue of (a), we have $\varphi_{\mathcal{B}}\left(z_{0}^{\prime}\right)=\varphi_{\mathcal{B}}\left(z_{m}\right)$. Therefore, it follows from the relation $\varphi_{\mathcal{B}}\left(z_{0}^{\prime}\right) \neq \varphi_{\mathcal{B}}\left(x_{0}^{\prime}\right)$ that $\varphi_{\mathcal{B}}\left(z_{m}\right) \neq \varphi_{\mathcal{B}}\left(x_{n}\right)$ and

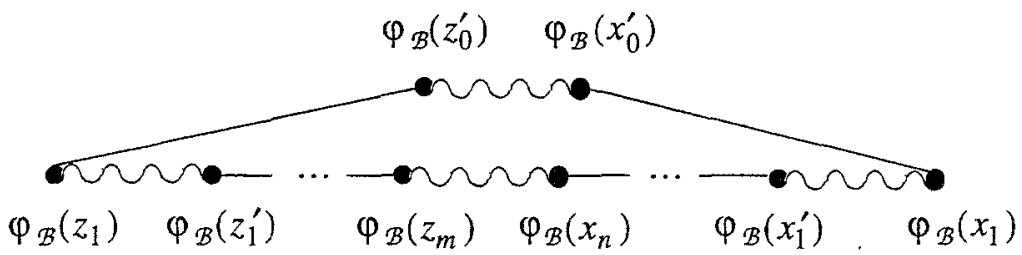

is an $S$-graph, which contradicts the assumption that $S$ is acyclic.

Lemma 8. If $S$ is acyclic, the $S$-graph $\mathcal{B}$ is connected, and $K(\mathcal{B})$ contains at least three vertices, then there exists a node $X \in K(\mathcal{B})$ such that the following relations hold:

(a) $\varphi_{\mathcal{B}}(X) \neq \varphi_{\mathcal{B}}(Y)$ for an arbitrary node $Y \in K(\mathcal{B})$,

(b) X contains at most one nondegenerate vertex.

Proof. (a) Assume that $\mathcal{A}=\left(A^{1}, \ldots, A^{m}\right)$ is the set of all classes from $S / \approx$ such that there exists a node $Y \in K(\mathcal{B})$ with $\varphi_{\mathcal{B}}(Y) \subset A^{i}$ for a proper $i=\overline{1, m}$. Let us transform $\mathcal{A}$ into an oriented graph. For this purpose, we assume that the arrow $A^{i} \rightarrow A^{j}$ exists if there are nodes $X_{i}, Y_{i}$, and $Z_{j}$ in $\mathcal{B}$ such that $\varphi_{\mathcal{B}}\left(X_{i}\right) \subset A^{i}$, $\varphi_{\mathcal{B}}\left(Y_{i}\right) \subset A^{i}, \varphi_{\mathcal{B}}\left(Z_{j}\right) \subset A^{j}$, and the path from $X_{i}$ to $Y_{i}\left[\right.$ in $K(\mathcal{B})$ ] passes through $Z_{j}$.

Let us prove that $\mathcal{A}$ contains no oriented cycles. Indeed, assume that, after a proper enumeration, it contains the cycle

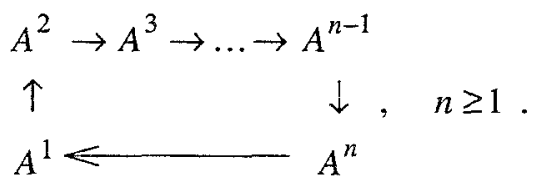

This means that, for every $i=\overline{1, n}$, there exists a path $\left(s_{i}\left|\mathbf{a}_{i}\right| t_{i}, p_{i}\left|\mathbf{b}_{i}\right| r_{i}\right)$ in the $S$-graph $\mathcal{B}$ with $\varphi_{\mathcal{B}}\left(s_{i}\right)$, 
$\varphi_{\mathcal{B}}\left(r_{i}\right) \in A^{i}, \varphi_{\mathcal{B}}\left(t_{i}\right), \varphi_{\mathcal{B}}\left(p_{i}\right) \in A^{i^{\prime}}$, and $t_{i} \sim p_{i}$ in $\mathcal{B}$; here, $\mathbf{a}_{i}$ and $\mathbf{b}_{i}$ are paths in $\mathcal{B}$, the symbol $\mid$ denotes linking of paths, and

$$
i^{\prime}=\left\{\begin{array}{cl}
i+1, & i=\overline{1, n-1} \\
1, & i=n
\end{array}\right.
$$

By virtue of Lemma $7, \varphi_{\mathcal{B}}\left(s_{i}\right)=\varphi_{\mathcal{B}}\left(r_{i}\right)$. By the definition of a path, $\varphi_{\mathcal{B}}\left(t_{i}\right) \sim \varphi_{\mathcal{B}}\left(p_{i}\right)$. Let

$$
\left(u_{i}\left|\mathbf{c}_{i}\right| v_{i}\right)= \begin{cases}\left(s_{i}\left|\mathbf{a}_{i}\right| t_{i}\right) & \text { if } \varphi_{\mathcal{B}}\left(t_{i}\right) \neq \varphi_{\mathcal{B}}\left(s_{i^{\prime}}\right), \\ \left(r_{i}\left|\overline{\mathbf{b}}_{i}\right| p_{i}\right) & \text { if } \varphi_{\mathcal{B}}\left(t_{i}\right)=\varphi_{\mathcal{B}}\left(s_{i^{\prime}}\right) .\end{cases}
$$

Here, $\overline{\mathbf{b}}$ is the path $\mathbf{b}$ passed in the opposite direction. Then

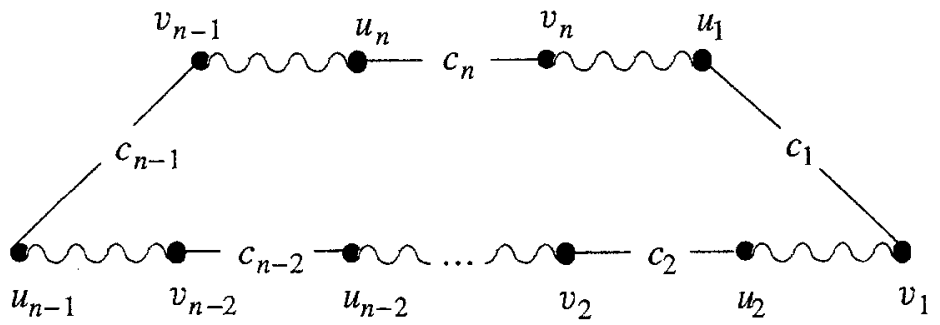

is an $S$-graph, which contradicts the assumption that $S$ is acyclic.

Thus, either $\mathcal{A}$ is a disconnected union of points or $\mathcal{A}$ contains at least one sink (i.e., a point with no outgoing arrows) with an arrow really entering this point. In the first case, the number of nondegenerate vertices of a node is not less than the number of edges of $K(\mathcal{B})$ originating from this node, and two nodes from this equivalence class in $S$ cannot be joined by an edge [in $K(\mathcal{B})$ ]. Therefore, as the required node $X$, one can take any node such that at least two edges originate from it.

In the second case, we assume that $B \in \mathcal{A}$ is a sink, $A \in \mathcal{A}$, and $A \rightarrow B$. Then, in $K(\mathcal{B})$, there exists a path of the form $Y_{1}-\ldots-X-\ldots-Y_{2}$, where $Y_{1}$ and $Y_{2}$ belong to $A, X$ belongs to $B$, and $X$ contains at least two vertices. If $X^{\prime} \in K(\mathcal{B})$ and $X^{\prime} \neq X$ is a node that also belongs to $B$, then it cannot be joined with $X$ by a straight line in $K(\mathcal{B})$ and there is a node between $X^{\prime}$ and $X$ that does not belong to $B$. Hence, $B$ is not a sink. Thus, $X$ is the required node.

Proposition 3. A completed biordered set $S$ is finite and acyclic if and only if $|\hat{C}(S)|<\infty$.

Proof. The necessity is obvious. To prove the proposition it suffices to show that, for a finite acyclic biordered set $S$, we have $\sup \left\{|\Gamma| \mid\left(\mathcal{B}, \Gamma,-, \sim, \varphi_{\mathcal{B}}\right) \in \hat{C}(S)\right\}<\infty$. For this purpose, we define functions $\bar{\varphi}_{\mathcal{B}}$ : $\mathcal{B} \rightarrow S / \approx$ and $\bar{\varphi}_{\mathcal{B}}=$ can $\circ \varphi_{\mathcal{B}}$ and note the following: By virtue of Lemma 7 , for any path $\left(x_{0}^{\prime}, x_{1}, \ldots, x_{n}\right)$ in the acyclic $S$-graph $\mathcal{B}$ and for any $i=\overline{1, n}$, there exists at most one $j=\overline{1, n}$ such that $\bar{\varphi}_{\mathcal{B}}\left(x_{i}\right)=\bar{\varphi}_{\mathcal{B}}\left(x_{j}\right)$. Therefore, the length of a path that joins two arbitrary vertices of $K(\mathcal{B})$ does not exceed $2|S / \approx|$. Since $K(\mathcal{B})$ is a tree, $|\Gamma / \approx|$ does not exceed the number of vertices of a complete tree of height $2|S| \approx \mid$ on the set $S \mid \approx$. This immediately implies that the value $|\Gamma|$ is bounded. 


\section{Elementary Representations}

For a chain vectroid $\mathcal{V}$, we set $S=S(\mathcal{V})$ and $\hat{C}(\mathcal{V})=\hat{C}(S(\mathcal{V}))$. A vectroid $\mathcal{V}$ is called quasifinite if $\hat{C}(\mathcal{V})$ is a finite set and def $\mathcal{V} \leq 1$. Below, we show that finitely represented vectroids are quasifinite.

We fix triangular bases $\left(m_{i}^{X}\right)$ of objects $X$ of the vectroid $\mathcal{V}$ and identify $s \in S(\mathcal{V})$ with the corresponding vector.

A representation $M$ of a locally finite completed biordered set $S$ is called elementary if the following conditions are satisfied:

(i) the elements of the matrix $M$ are equal to either 0 or 1 ;

(ii) every row of $M$ contains at most two unit entries; each of its columns contains at most one unit entry;

(iii) if there are two nonzero elements in a row of $M$, they belong to incomparable columns.

In particular, by setting $S=S(\mathcal{V})$, we arrive at the notion of elementary representation of the vectroid $\mathcal{V}$ [in the basis $\left.\left(m_{i}^{X}\right)\right]$.

For every matrix of elementary representation nondegenerate with respect to rows, we construct an $S(\mathcal{V})$ graph as follows:

(i) vertices of the graph are considered as columns of the matrix $M$ of the given representation;

(ii) nonzero columns of the matrix $M$ are regarded as nondegenerate vertices;

(iii) above each vertex, we write the element of $S(\mathcal{V})$ related to the corresponding column;

(iv) we join two vertices by a straight line if these columns have a unit entry in a common row;

(v) we join two vertices by a wavy line if these columns are equivalent.

Conversely, an arbitrary $S$-graph $\mathcal{B} \in \hat{C}(\mathcal{V})$ is associated with a nondegenerate elementary representation $R(\mathcal{B}) \in \operatorname{Rep} \mathcal{V}$. For a node $X \in K(\mathcal{B})$, we denote by $V_{X}$ a uniquely defined object of the vectroid $\mathcal{V}$ such that $\varphi_{\mathcal{B}}(X) \subset V_{X}$. Let

$$
X_{\mathcal{B}}=\underset{X \in K(\mathcal{B})}{\oplus} V_{X},
$$

let $U_{\mathcal{B}}$ be a subspace of $X_{\mathcal{B}}$ spanned either on the vectors $\varphi_{\mathcal{B}}(x)$ if $x \in \Gamma$ and $\Gamma^{-}(x)=\varnothing$ or on the vectors $\varphi_{\mathcal{B}}(x)+\varphi_{\mathcal{B}}(y) \in V_{\mathcal{B}^{\sim}(x)} \oplus V_{\mathcal{B}}(y)$ if $x-y$ in $\Gamma$, and let $i_{\dot{B}} U_{\mathcal{B}} \rightarrow X_{\mathcal{B}}$ be the imbedding of subspaces. Thus, we obtain the representation $R(\mathcal{B})=\left(U_{\mathcal{B}}, i_{\mathfrak{B}}, X_{\mathcal{B}}\right)$, which has the matrix required in the definition of elementary representations in the basis $S(\mathcal{V})$ of the vectroid $\mathcal{V}$ and in the basis $U_{\mathcal{B}}$ formed of the generating vectors. Furthermore, $R(\mathcal{B}) \in{ }^{i} \operatorname{Rep} V$ because $i_{\mathcal{B}}$ is an injection. In what follows, we identify a vertex $x$ of the $S$-graph $\mathcal{B}$ and the vector $\varphi_{\mathcal{B}}(x) \in V_{\mathcal{B}^{\approx}(x)}$. Note that $\{x \mid x \in \mathcal{B}\}$ is a basis of the space $X_{\mathcal{B}}$.

Note that $R$ establishes a bijection between the set of classes of isomorphisms of $S(\mathcal{V})$-graphs and the set of matrices of elementary representations nondegenerate with respect to the rows of the vectroid $\mathcal{V}$ in the given basis $S(\mathcal{V})$; here, the matrices are considered up to permutations of rows and columns. 
In view of the structure of the bijection $R$, we can assume that every node $\mathcal{B}^{\approx}(x)$ of the $S$-graph $\mathcal{B}$ consists of elements of the set $S \times \mathbb{N}$ of the form $(s, i)$, where $s \in S^{\approx}\left(\varphi_{\mathcal{B}}(x)\right)$ and $i$ is the number of the corresponding columns in bands determined by elements $s$ of a certain matrix of the representation $R(\mathcal{B})$.

Example 3. Generally speaking, an elementary representation in one basis can be equivalent to a nonelementary representation in another basis. Let $\mathcal{W}$ be the vectroid defined in Example 2 and let $\left(\begin{array}{llll}1 & 0 & 0 & 0 \\ 0 & 0 & 1 & 0\end{array}\right)$ be the matrix of a representation of $\mathcal{W}$. In this case, the representation is elementary in the basis $m_{1}^{X}, m_{2}^{X}, m_{3}^{X}, m_{4}^{X}$. However, in the basis $\bar{m}_{1}^{X}=m_{1}^{X}-m_{2}^{X}, \bar{m}_{2}^{X}=m_{2}^{X}, \bar{m}_{3}^{X}=m_{3}^{X}, \bar{m}_{4}^{X}=m_{4}^{X}$, it is determined by the matrix $\left(\begin{array}{llll}1 & 1 & 0 & 0 \\ 0 & 0 & 1 & 0\end{array}\right)$, which is not equivalent to an elementary one.

Let $\mathcal{V}_{1}$ and $\mathcal{V}_{2}$ be two chain vectroids, $S_{1}=S\left(\mathcal{V}_{1}\right), S_{2}=S\left(\mathcal{V}_{2}\right)$, and let $f$ be an injective map $S_{1} \rightarrow S_{2}$ (which does not take into account structures on $S_{1}$ and $S_{2}$ ). Assume that $f$ is induced by a morphism $(F, \Phi) \in$ $\mathcal{M}\left(\mathcal{V}_{1}, \mathcal{V}_{2}\right)$

For a nondegenerate $S_{1}$-graph $\left(\mathcal{B}, \Gamma,-, \sim, \varphi_{\mathcal{B}}\right)$, we define a nondegenerate $S_{2}$-graph $\left(\mathcal{D}, \Delta,-^{\prime}, \sim^{\prime}, \varphi_{\mathcal{D}}\right)$ as follows: $\Delta=\Gamma$ and, for $x, y \in \Delta$,

(i) $x-^{\prime} y$ if $x-y$ (in $\Gamma$ ) and $f \circ \varphi_{\mathcal{B}}(x) \ngtr f \circ \varphi_{\mathcal{B}}(y)\left(\right.$ in $\left.S_{2}\right)$;

(ii) $x \sim^{\prime} y$ if $x \sim y$ (in $\Gamma$ ) and $f \circ \varphi_{\mathcal{B}}(x) \sim f \circ \varphi_{\mathcal{B}}(y)$ (in $S_{2}$ );

(iii) $\left.\varphi_{\mathcal{D}}\right|_{\Delta}=\left.f \circ \varphi_{\mathcal{B}}\right|_{\Gamma}$.

Then we reconstruct $\mathcal{D}$ by using Remark 6 . Obviously, $f_{*}(\mathcal{B})$ is a nondegenerate $S_{2}$-graph.

Let $f$ be such that, for $s, t \in S_{1}$, it follows from $s \nless t$ that $f(s) \preccurlyeq f(t)$. Then, clearly, $\operatorname{Der}(F, \Phi)(R(\mathcal{B}))=$ $R\left(f_{*}(\mathcal{B})\right)$.

Example 4. Let $\mathcal{B}$ be a nondegenerate $S$-graph.

(a) Let $\mathcal{V}$ be a vectroid, $S=S(\mathcal{V})$, let $S^{w}=(S, \leq, \approx)$ be a weakly completed poset (Sec. 1), and let $w^{s}: S \rightarrow S^{w}$ be an identity map of supporting sets. By using $S^{w}$, we construct a vectroid $\mathcal{V}^{w}$ (whose rank does not exceed one) and obtain a morphism $W \in \mathcal{M}\left(\mathcal{V}, \mathcal{V}^{w}\right)$ from $w^{s}$. Then $R\left(w_{*}^{s}(\mathcal{B})\right)=$ $\operatorname{Der}(W)(R(\mathcal{B}))$.

(b) For a weakly completed poset $(S, \leq, \approx)$ and $Q \subset S$, we construct $S^{Q}=\left(S, \leq, \approx^{\prime}\right)$; let $d^{Q}: S \rightarrow S^{Q}$ be an identity map and assume that $x \sim^{\prime} y$ if and only if $x \sim y$ and $x, y \notin Q$. The operation of passing from $S$ to $S^{Q}$ with the help of $d^{Q}$ (or a morphism of the corresponding vectroids $d^{Q}: \mathcal{V} \rightarrow \mathcal{V}^{Q}$ ) is called a wave break for $Q$. The following equality is true:

$$
R\left(d_{*}^{Q}(\mathcal{B})\right)=\operatorname{Der}\left(D^{Q}\right)(R(\mathcal{B}))
$$

Below, we obtain criteria for quasifinite vectroids to be indecomposable and for elementary representations to be equivalent. First, note that if an $S$-graph $\mathcal{B}$ is disconnected, then the representation $R(\mathcal{B})$ is decomposable. Indeed, let $\mathcal{B}=\mathcal{B}_{1} \Perp \mathcal{B}_{2}$. In this case, $R(\mathcal{B})=R\left(\mathcal{B}_{1}\right) \oplus R\left(\mathcal{B}_{2}\right)$, which directly follows from the construction. 
Proposition 4. Suppose that a vectroid $\mathcal{V}$ is acyclic, $\mathcal{B}$ and $\mathcal{D}$ are $S$-graphs, and the $S$-graph $\mathcal{B}$ is connected. Then the following statements are true:

(a) If $R(\mathcal{B}) \simeq R(\mathcal{D})$, then $\mathcal{B}$ is isomorphic to $\mathcal{D}$.

(b) $R(\mathcal{B})$ is indecomposable.

Proof. First, note that Example 4(a) allows us to regard $S$ as a weakly completed poset. We prove the proposition by induction on $\mathbf{d}(\mathcal{B})$ for all weakly competed posets $S$ and $S$-graphs $\mathcal{B}$ simultaneously. Denote by $\mathbf{d}(\mathcal{B})$ the number of waves in $\Gamma$, i.e., $\mathbf{d}(\mathcal{B})=|\{\{x, y\} \subseteq \Gamma \mid x \sim y\}|$. In this case, if there is a node in $\mathcal{B}$ that contains at least two nondegenerate vertices and one of them belongs to an element of $Q$, then $\mathbf{d}\left(d_{*}^{Q}(\mathcal{B})\right)<\mathbf{d}(\mathcal{B})$. If $\Gamma=\varnothing$, the proposition is obvious (see Remark 6). Therefore, we assume that $\Gamma \neq \varnothing$.

We take $\mathbf{d}(\mathcal{B})=0$ as a basis of induction. By virtue of connectedness, $\mathcal{B}$ has the form

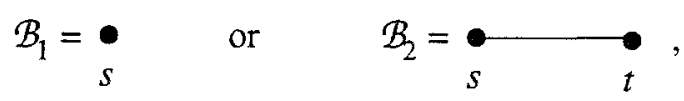

where $s, t \in S$. In this case, the proposition can easily be proved by using the fact that $S$ is acyclic and the exchange theorem ([1], 3.3, b).

Induction step. By virtue of Lemma 8 , there are two possibilities for the connected $S$-graph $\mathcal{B}$, namely, either it contains no vertices joined by wavy lines, i.e., $\mathbf{d}(\mathcal{B})=0$, or there is a node containing at least two vertices and there is no other node in $\mathcal{B}$ that belongs to the same equivalence class in $S$. By choosing an arbitrary vertex $x$ from this node and setting $Q=\left\{\varphi_{\mathcal{B}}(x)\right\}$, we get $\mathcal{B}^{\prime}=d_{*}^{Q}(\mathcal{B})$ and $\mathcal{D}^{\prime}=d_{*}^{Q}(\mathcal{D})$, where $\mathcal{B}^{\prime}=\mathcal{B}_{1} \Perp \mathcal{B}_{2}, \mathcal{B}_{1}$ and $\mathcal{B}_{2}$ are connected $S\left(\mathcal{V}^{Q}\right)$-graphs, and $R\left(\mathcal{B}^{\prime}\right) \simeq R\left(\mathcal{D}^{\prime}\right)$ [see Example 4(b)].

By the induction hypothesis and the uniqueness of the decomposition in direct sum in $\operatorname{Rep} \mathcal{V}$, we establish that $\mathcal{D}^{\prime}=\mathcal{B}^{\prime}=\mathcal{B}_{1} \Perp \mathcal{B}_{2}, R\left(\mathcal{D}^{\prime}\right)=R\left(\mathcal{B}_{1}\right) \oplus R\left(\mathcal{B}_{2}\right)$, and $R\left(\mathcal{B}_{1}\right)$ and $R\left(\mathcal{B}_{2}\right)$ are indecomposable. Therefore, either $\mathcal{D}=\mathcal{B}$ or $\mathcal{D}=\mathcal{B}_{1} \Perp \mathcal{B}_{2}$. In the latter case, $X_{\mathcal{D}}$ contains two direct summands of the form $V_{\mathcal{B}} \approx(x)$ and $X_{\mathcal{B}}$ contains one such summand, which is impossible. If $R(\mathcal{B})$ is decomposable, i.e., $R(\mathcal{B})=\left(U_{1}, i_{1}, I_{1}\right) \oplus\left(U_{2}, i_{2}\right.$, $\left.I_{2}\right)$, then $\operatorname{Der}\left(\mathcal{D}^{Q}\right)\left(U_{1}, i_{1}, I_{1}\right) \simeq R\left(\mathcal{B}_{1}\right)$ and $\operatorname{Der}\left(\mathcal{D}^{Q}\right)\left(U_{2}, i_{2}, I_{2}\right) \simeq R\left(\mathcal{B}_{2}\right)$. We again get two direct summands of the form $V_{\mathcal{B}^{\approx}(x)}$ in $I_{1} \oplus I_{2}$, but $X_{\mathcal{B}}$ has one such summand. Thus, $R(\mathcal{B})$ is indecomposable. Proposition 4 is proved.

Corollary 1. Let a vectroid $\mathcal{V}$ be acyclic and let $\mathcal{B}$ and $\mathcal{D}$ be two arbitrary $S(\mathcal{V})$-graphs. Then the relation $\mathcal{B} \simeq \mathcal{D}$ follows from $R(\mathcal{B}) \simeq R(\mathcal{D})$.

This statement is a consequence of Proposition 4 (a) and Remark 6.

Example 5. Let $\mathcal{V}$ be a chain vectroid and let $S(\mathcal{V})$ be not acyclic. Let us prove that $\mathcal{V}$ has infinitely many nonisomorphic indecomposable elementary representations.

Assume that the nondegenerate $S=S(\mathcal{V})$-graph

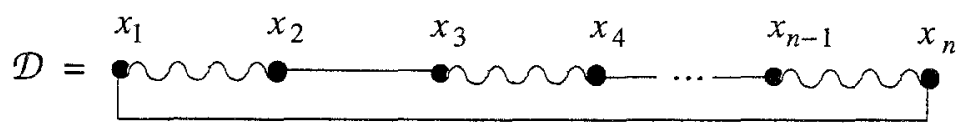

is a cycle (note that $n>3$ ). We assume that the cycle $\mathcal{D}$ is minimal. We define the $S$-graph 


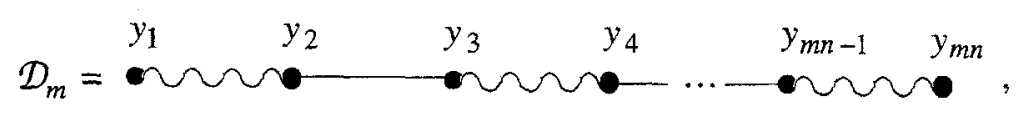

$\varphi_{\mathcal{D}_{m}}\left(y_{i}\right)=\varphi_{\mathcal{D}}\left(x_{\tilde{i}}\right)$, where $1 \leq \tilde{i} \leq n$ and $\tilde{i} \bmod n=i \bmod n$. Then, for any $m \in \mathbb{N}$, the representation $R\left(\mathcal{D}_{m}\right) \in$ Rep $\mathcal{V}$ is indecomposable. In this case, obviously, $R\left(\mathcal{D}_{m}\right) \neq R\left(\mathcal{D}_{m^{\prime}}\right)$ if $m \neq m^{\prime}$. Example 4 (a) allows us to assume that $S$ is a weakly completed poset.

For a vertex $y$ of the $S$-graph $\mathcal{D}_{m}$, we denote by $y^{\prime}$ the nondegenerate vertex such that $y^{\prime}-y$. For $y \notin$ $\left\{y_{1}, y_{m_{n}}\right\}$, we denote by $\bar{y}$ the vertex such that $\bar{y}-y$.

Let $M$ be the matrix of the representation $R\left(\mathcal{D}_{m}\right), \operatorname{dim} U_{D_{m}}=l, \operatorname{dim} X_{D_{m}}=k$. Let us introduce a partial ordering $\preceq$ on the set of nondegenerate vertices $\Delta_{m}$ of the $S$-graph $\mathcal{D}_{m}$. Let $y_{i}$ and $y_{j}$ belong to $\Delta_{m}$. In the case $\varphi_{\mathcal{D}_{m}}\left(y_{i}\right) \neq \varphi_{\mathcal{D}_{m}}\left(y_{j}\right)$, we set $y_{i} \prec y_{j}$ if and only if $\varphi_{\mathcal{D}_{m}}\left(y_{i}\right)<\varphi_{\mathcal{D}_{m}}\left(y_{j}\right)$. If $\varphi_{\mathcal{D}_{m}}\left(y_{i}\right)=\varphi_{\mathcal{D}_{m}}\left(y_{j}\right)$, then, in order to define $\prec$, we construct the following two sequences of integer pairs (possibly, of zero length):

$$
\begin{aligned}
& \left(a_{1}, b_{1}\right), \ldots,\left(a_{\alpha}, b_{\alpha}\right),\left(a_{\alpha+1}, b_{\alpha+1}\right), \ldots, \\
& \left(c_{1}, d_{1}\right), \ldots,\left(c_{\alpha}, d_{\alpha}\right),\left(c_{\alpha+1}, d_{\alpha+1}\right), \ldots
\end{aligned}
$$

If $j \in\{1, m n\}$, we set $y_{i} \prec y_{j}$ and do not start the construction; if $i \in\{1, m n\}$, we set $y_{i} \nprec y_{j}$ and also do not start the construction. If $i, j \notin\{1, m n\}$, then $\left(y_{a_{1}}, y_{b_{1}}\right)=\left(y_{i}, y_{j}\right)$ and $\left(y_{c_{1}}, y_{d_{1}}\right)=\left(\bar{y}_{i}, \bar{y}_{j}\right)$.

We continue the construction by induction. If $\left(a_{\alpha}, b_{\alpha}\right)=\left(c_{\alpha}, d_{\alpha}\right)=(0,0)$, then the construction is completed and we set $y_{i} \prec y_{j}$. If $\left(a_{\alpha}, b_{\alpha}\right)=(0,0)$, then $\left(a_{\alpha+1}, b_{\alpha+1}\right)=(0,0)$. If $\left(c_{\alpha}, d_{\alpha}\right)=(0,0)$, then $\left(c_{\alpha+1}, d_{\alpha+1}\right)=(0,0)$. If $y_{a_{\alpha}}^{\prime}$ or $y_{c_{\alpha}}^{\prime}$ belongs to $\left\{y_{1}, y_{m n}\right\}$, then the construction is completed and we set $y_{i} \nprec y_{j}$. If $b_{\alpha} \in\{1, m n\}$, then $\left(a_{\alpha+1}, b_{\alpha+1}\right)=(0,0)$. If $d_{\alpha} \in\{1, m n\}$, then $\left(c_{\alpha+1}, d_{\alpha+1}\right)=(0,0)$. Otherwise, if $\left(a_{\alpha}, b_{\alpha}\right) \neq(0,0)$ and $y_{a_{\alpha}}^{\prime}, y_{b_{\alpha}}^{\prime} \neq y_{1}, y_{m n}$, then $\left(y_{a_{\alpha+1}}, y_{b_{\alpha+1}}\right)=\left(\left(y_{a_{\alpha}}^{\prime}\right)^{-},\left(y_{b_{\alpha}}^{\prime}\right)^{-}\right)$. If $\left(c_{\alpha}, d_{\alpha}\right) \neq$ $(0,0)$ and $y_{c_{\alpha}}^{\prime}, y_{d_{\alpha}}^{\prime} \neq y_{1}, y_{m n}$, then

$$
\left(y_{c_{\alpha+1}}, y_{d_{\alpha+1}}\right)=\left(\left(y_{c_{\alpha}}^{\prime}\right)^{-},\left(y_{d_{\alpha}}^{\prime}\right)^{-}\right) .
$$

By construction, the relation $y_{i} \preceq y_{j} \preceq y_{i}$ implies that $y_{i}=y_{j}$.

Let us introduce a partial ordering $\sqsubseteq$ on rows of $M$. For this purpose, note that the set of rows of $M$ is bijective to the set $K\left(\mathcal{D}_{m}\right)=\left\{\left\{y_{1}\right\},\left\{y_{2}, y_{3}\right\}, \ldots,\left\{y_{m n 2}, y_{m+1}\right\},\left\{y_{m n}\right\}\right\}$. We set $A \sqsubseteq B, A, B \in K\left(\mathcal{D}_{m}\right)$, if, for every $z \in B$, one can find $\tilde{z} \in A$ such that $\tilde{z} \preceq z$ (cf. [5, p. 13]).

Let $(\varphi, \xi) \in \operatorname{End}_{\operatorname{Rep} \mathcal{V}}\left(R\left(\mathcal{D}_{m}\right)\right)$ be an endomorphism and let $\left(F=\left(f_{i j}\right), G=\left(g_{i j}\right)\right)$ be its matrix notation. In particular, $F M=M G$. Then, by analogy with the proof of Lemma 10, we can show that $f_{i j} \neq 0$ for some $(\varphi, \xi)$ if and only if $i \sqsubseteq j, i, j=\overline{1, l}$, and $g_{i j} \neq 0$ for some $(\varphi, \xi)$ if and only if $i \preceq j, i, j=\overline{1, k}$. Hence, we conclude that $\sqsubseteq$ and $\preceq$ are transitive and, therefore, they are orders.

Let us introduce linear orders on the bases $U_{\mathcal{D}_{m}}$ and $X_{\mathcal{D}_{m}}$ so that $i \sqsubseteq j$ yields $i \leq j$ (for $U_{\mathcal{D}_{m}}$ ) and $i \preceq j$ yields $i \leq j$ (for $\chi_{\mathcal{D}_{m}}$ ). This can be done because every order can be extended to a linear one (see [14], VII.8, Theorem 8 ). For such an ordering of bases, the matrices $F$ and $G$ are upper triangular.

Assuming that $R\left(\mathcal{D}_{m}\right)$ is decomposable, we can find an idempotent $(\varphi, \xi)$. Then, for arbitrary $\lambda \in k^{*}$, the pair $\left(\varphi+\lambda \mathrm{id}_{U_{\mathcal{D}_{m}}}, \xi+\lambda \mathrm{id}_{X_{\mathcal{D}_{m}}}\right)$ is an endomorphism of $R\left(\mathcal{D}_{m}\right)$. An arbitrary nonzero element of the matrix $(F+$ 
$\left.\lambda E_{l}\right) M$ has the form $\left(\lambda+f_{i i}\right) m_{i t}$ or $\sum_{j \neq i} a_{i j} m_{j t}$. Similarly, every nonzero element of the matrix $M(G+$ $\left.\lambda E_{k}\right)$ has the form $m_{i t}\left(\lambda+g_{t t}\right)$ or $\sum_{j \neq i} m_{i j} g_{j t}$. Since $\left(\lambda E_{l}+F\right) M=M\left(\lambda E_{k}+G\right)$ for all $\lambda \in k^{*}$, the nonzero element of this matrix is equal to $\left(\lambda+f_{i i}\right) m_{i t}=m_{i t}\left(\dot{\lambda}+g_{t t}\right)$ or $\sum_{j \neq i} a_{i j} m_{j t}=\sum_{j \neq i} m_{i j} g_{j t}$. Therefore, $(\bar{\varphi}, \bar{\xi}) \in \operatorname{End}\left(R\left(\mathcal{D}_{m}\right)\right.$ ) (here, $\bar{\varphi}$ is the diagonal of the matrix $\varphi$ ).

But then we can conclude that the $S$-graph $\mathcal{D}_{m}$ is disconnected, which is not true. Therefore, the representation $R\left(\mathcal{D}_{m}\right)$ is indecomposable.

Proposition 5. Every chain vectroid $\mathcal{V}$ has finitely many classes of isomorphisms of indecomposable elementary representations if and only if $|\hat{C}(\mathcal{V})|<\infty$.

This statement obviously follows from Propositions 3 and 4 and Example 5.

Corollary 2. A finitely represented vectroid $\mathcal{V}$ is quasifinite.

The validity of this statement follows from Proposition 5.

\section{Order on $C(S)$}

A pair $(\mathcal{B}, x)$, where $\mathcal{B}$ is a connected $S$-graph and $x \in \mathcal{B} \backslash \Gamma$, is called an $S$-graph with marked vertex. Denote the set of classes of isomorphisms of $S$-graphs with marked vertex by $C(S)$. We assume that the completed biordered set $S$ is acyclic.

Let $\left(\mathcal{B}, \Gamma_{2}-, \sim, \varphi_{\mathcal{B}}\right)$ be a connected $S$-graph and let $x \in \mathcal{B}$ be its vertex. We define (connected) $S$-graphs $l_{x} \mathcal{B}$ and $r_{x} \mathcal{B}$ as follows:

(a) Let $x \in \Gamma$ and let $\Gamma^{-}(x)=\{y\}$. We remove the straight line that joins $x$ and $y$ from $\Gamma$, i.e., we introduce a relation $-^{\prime}$ on $\Gamma$ as follows: For $z_{1}, z_{2} \in \Gamma$, we have $z_{1}-z_{2}^{\prime}$ if and only if $z_{1}-z_{2}$ and $\left\{z_{1}, z_{2}\right\} \neq\{x, y\}$. Then, since $S$ is acyclic, $\left(\mathcal{B}, \Gamma \backslash\{x, y\},-^{\prime},-, \varphi_{\mathcal{B}}\right)$ is a disconnected $S$-graph with two connected components. One of these, denoted by $l_{x} \mathcal{B}$, contains the vertex $x$, and the other one, denoted by $r_{x} \mathcal{B}$, contains $y$.

(b) Let $x \in \Gamma$ and let $\Gamma^{-}(x)=\varnothing$. In this case, $l_{x} \mathcal{B}=\left(\mathcal{B}, \Gamma \backslash\{x\},-^{\prime}, \sim, \varphi_{\mathcal{B}}\right)$, where -' is a restriction to $\Gamma \backslash\{x\}$ and $r_{x} \mathcal{B}=\left(\mathcal{B}^{\approx}(x), \varnothing,\left.\sim\right|_{\mathcal{B}^{\approx}(x)},\left.\varphi_{\mathcal{B}}\right|_{\mathcal{B}^{\approx}(x)}\right)$.

(c) Let $x \notin \Gamma$. In this case, $l_{x} \mathcal{B}=\mathcal{B}$ and $r_{x} \mathcal{B}=\varnothing$.

For $(\mathcal{B}, x) \in C(S)$ and $s \in S^{\sim}\left(\varphi_{\mathcal{B}}(x)\right)$, we now introduce derivatives $\partial_{s}^{1}(\mathcal{B}, x)$ and $\partial_{s}^{2}(\mathcal{B}, x)$. Let $y \in \mathcal{B}^{\sim}(x)$ and let $\varphi_{\mathcal{B}}(y)=s$. We set

$$
\begin{gathered}
\partial_{s}^{1}(\mathcal{B}, x)=\left(l_{y} \mathcal{B}, y\right), \\
\partial_{s}^{2}(\mathcal{B}, x)=\left\{\begin{array}{cl}
\left(r_{y} \mathcal{B}, z\right) & \text { if } y \in \Gamma \text { and } y-z ; \\
0 & \text { if } y \notin \Gamma ; \\
1 & \text { if } y \in \Gamma, \Gamma^{-}(y)=\varnothing .
\end{array}\right.
\end{gathered}
$$


We denote $\partial_{x^{\prime}}(\mathcal{B}, x)=\partial_{\varphi_{\mathcal{B}}\left(x^{\prime}\right)}(\mathcal{B}, x)$ for a vertex $x^{\prime} \in \mathcal{B}^{\sim}(x)$. For an $S$-graph $(\mathcal{D}, y)$ with $\varphi_{\mathcal{D}}(y) \approx \varphi_{\mathcal{B}}(x)$ and for a vertex $y^{\prime} \in \mathcal{D}^{\approx}(y), \varphi_{\mathcal{D}}\left(y^{\prime}\right) \sim \varphi_{\mathcal{B}}(x)$, we write $\partial_{y^{\prime}}(\mathcal{B}, x)=\partial_{\varphi_{\mathcal{D}}\left(y^{\prime}\right)}(\mathcal{B}, x)$.

Let us introduce a relation $\leq$ on $C(S)$. First, we add the maximal element 1 and the minimal element 0 to $C(S)$ (the relation $\leq$ on $C(S)$ is induced by the relation $\leq$ on $C(S) \Perp\{0,1\}$ ). Consider the $S$-graphs $(\mathcal{B}, x)$ and $(\mathcal{D}, y) \in C(S)$. The relation $\leq$ is defined by induction on the common number of vertices of both $S$-graphs $\mathcal{B}$ and $\mathcal{D}$.

(a) If $\varphi_{\mathcal{B}}(x) \nless \varphi_{\mathcal{D}}(y)$, we set $(\mathcal{B}, x) \preccurlyeq(\mathcal{D}, y)$;

(b) If $\varphi_{\mathcal{B}}(x) \triangleleft \varphi_{\mathcal{D}}(y)$, we set $(\mathcal{B}, x) \leq(\mathcal{D}, y)$;

(c) If $\varphi_{\mathcal{B}}(x)=\varphi_{\mathcal{D}}(y)=s$ and $s \rtimes s$, then $(\mathcal{B}, x) \leq(\mathcal{D}, y)$ if and only if $\partial_{t}^{2}(\mathcal{B}, x) \leq \partial_{t}^{2}(\mathcal{D}, y)$ for every $t \in S^{-}(s)$;

(d) If $s_{1}=\varphi_{\mathcal{B}}(x) \Rightarrow s_{2}=\varphi_{\mathcal{D}}(y)$, then $(\mathcal{B}, x) \leq(\mathcal{D}, y)$ if and only if there exist $t_{1}, t_{2} \in S$ such that $s_{1} \Rightarrow s_{2}, t_{1} \Rightarrow t_{2}, s_{1} \sim t_{1}, s_{2} \sim t_{2}$, and at least one of the following conditions is satisfied:

$$
\begin{aligned}
& \partial_{t_{1}}^{2}(\mathcal{B}, x) \leq \partial_{t_{2}}^{2}(\mathcal{D}, y) \\
& \partial_{t_{1}}^{1}(\mathcal{B}, x) \leq \partial_{t_{2}}^{2}(\mathcal{D}, y) \\
& \partial_{t_{1}}^{2}(\mathcal{B}, x) \leq \partial_{t_{2}}^{1}(\mathcal{D}, y) .
\end{aligned}
$$

The reason for introducing the relation $\leq$ is clarified by the following construction: Let $\mathcal{V}$ be a chain vectroid and let $S=S(\mathcal{V})$. We define a spectroid El of elementary representations with marked zero column as follows: The objects of the spectroid $\mathrm{El}$ are the elements of the set $C(\mathcal{V})=C(S(\mathcal{V}))$. For $(\mathcal{B}, x),(\mathcal{D}, y) \in C(\mathcal{V})=\mathrm{El}$, morphisms $(\varphi, \xi) \in \operatorname{Rep} \mathcal{V}(R \mathcal{B}, R \mathcal{D}))$ such that $x \xi \in U_{\mathcal{D}}+y k$ form the set of morphisms $\mathrm{El}((\mathcal{B}, x),(\mathcal{D}, y))$. Note that $y \notin U_{\mathcal{D}}$ and $U_{\mathcal{D}}+y k$ is a subspace of $X_{\mathfrak{D}}$. Obviously, $\mathrm{El}$ is a category because $\mathrm{El}((\mathcal{B}, x),(\mathcal{D}, y))$ 。 $\operatorname{El}((\mathcal{D}, y),(\mathcal{E}, z)) \subset \operatorname{El}((\mathcal{B}, x),(\mathcal{E}, z))$

Lemma 9. If a vectroid $\mathcal{V}$ is acyclic, then $\mathrm{El}$ is a spectroid.

Proof. The indecomposability of the representation $R(\mathcal{B})$ proved in Proposition 4 (b) implies that the object $(\mathcal{B}, x) \in \mathrm{El}$ is indecomposable.

Let us show that different objects of the category $\mathrm{El}$ are not isomorphic. Let $(\mathcal{B}, x),(\mathcal{D}, y) \in \mathrm{El}$. If $\mathcal{B} \neq \mathcal{D}$, then $R(\mathcal{B}) \neq R(\mathcal{D})$ by virtue of Proposition 4 (a). Hence, $(\mathcal{B}, x) \neq(\mathcal{D}, y)$. If $\mathcal{B}=\mathcal{D}$ but $x \neq y$, then the isomorphism $(\varphi, \xi):(\mathcal{B}, x) \rightarrow(\mathcal{D}, x)$ induces the isomorphism $(\varphi, \xi): R\left(\mathcal{B}^{\prime}\right) \simeq R\left(\mathcal{D}^{\prime}\right)$, where $\mathcal{B}^{\prime}=$ $\left(\mathcal{B}, \Gamma \cup\{x\},-,-, \varphi_{\mathcal{B}}\right)$ and $\mathcal{D}^{\prime}=\left(\mathcal{B}, \Gamma \cup\{y\},-, \sim, \varphi_{\mathcal{B}}\right)$. Recall that $\mathcal{B}=\mathcal{D}$ and $x, y \notin \Gamma$; the relation - on $\mathcal{B}^{\prime}$ and $\mathcal{D}^{\prime}$ is a trivially extended relation - on $\Gamma$. Thus, it suffices to prove that the $S$-graphs $\mathcal{B}^{\prime}$ and $\mathcal{D}^{\prime}$ are not isomorphic if $x \neq y$.

If $\mathcal{B}^{\prime} \simeq \mathcal{D}^{\prime}$, then $\varphi_{\mathcal{B}}(x)=\varphi_{\mathcal{B}}(y)$. By Lemma 8 , there exists a node $R$ in $K(\mathcal{B})$ such that $\varphi_{\mathcal{B}}(R) \approx \varphi_{\mathcal{B}}(Q)$ for any node $Q \in K(\Gamma)$. The connectedness of $\mathcal{B}$ implies that there exist paths $\left(x_{0}^{\prime}, x_{1}, x_{1}^{\prime}, \ldots, x_{n-1}, x_{n-1}^{\prime}, x_{n}\right)$ and $\left(y_{0}^{\prime}, y_{1}, y_{1}^{\prime}, \ldots, y_{m-1}, y_{m-1}^{\prime}, y_{m}\right)$ that join $\mathcal{B}^{\approx}(x)$ and $\mathcal{B}^{\approx}(y)$, respectively, with $R$ for which $x_{0}^{\prime} \approx x, y_{0}^{\prime} \approx y$, $x_{n} \in R$, and $y_{m} \in R$. 
If $f: \mathcal{B}^{\prime} \rightarrow \mathcal{D}^{\prime}$ is an isomorphism, then, for any $z \in R \cap \Gamma$, we have $f\left(r_{z} \mathcal{B}\right) \subset r_{z} \mathcal{D}$. This readily follows from the facts that $f \mid R$ is an identity and $f$ transforms the path joining some node with $R$ into the path that joins the image of this node with $R$. Therefore, $f$ induces the isomorphism $r_{z} \mathcal{B}^{\prime} \simeq r_{z} \mathcal{D}^{\prime}$ for every $z \in \Gamma \cap R$.

If $x_{n}=y_{m}$, then, passing to the the $S$-graph $x_{n} \mathcal{B}$, we can assume that $x_{n} \neq y_{m}$. If $x_{n} \neq y_{m}$, then, as proved above, we get $r_{y_{m}} \mathcal{B}^{\prime} \simeq r_{y_{m}} \mathcal{D}^{\prime}$, which is not true because $x$ is a nondegenerate vertex in $r_{y_{m}} \mathcal{D}^{\prime}$ and a degenerate vertex in $r_{y_{m}} \mathcal{B}^{\prime}$. Lemma 9 is proved.

There is a one-dimensional (nonfaithful) module $N, N(\mathcal{B}, x)=a_{\mathcal{B}, x} k\left(\simeq U_{\mathcal{B}}+x k / U_{\mathcal{B}}\right)$, over the category El. Every morphism $(\varphi, \xi) \in \operatorname{El}((\mathcal{B}, x),(\mathcal{D}, y))$ induces a linear map $\bar{\xi}: N(\mathcal{B}, x) \rightarrow N(\mathcal{D}, y)$, which establishes the structure of the El-module on $N$. The correctness of the definition is obvious.

We set $\overline{\mathrm{El}}=\mathrm{El} / \mathrm{Ann}_{\mathrm{El}} N$.

Lemma 10. Let a vectroid $\mathcal{V}$ be quasifinite. Then $\overline{\mathrm{El}}((\mathcal{B}, x),(\mathcal{D}, y)) \neq 0$ if and only if $(\mathcal{B}, x) \leq(\mathcal{D}, y)$.

Proof. 1. For the $S$-graph $\mathcal{B}$, we denote by $\left\{\tau_{z}, z \in \mathcal{B}\right\}$ the basis of the space $D X_{\mathcal{B}}\left(=\bmod k\left(\mathcal{X}_{\mathcal{B}}, k\right)\right)$ dual to the basis $\{z, z \in \mathcal{B}\}$. In this case, it follows from step I of the proof of Lemma 2 that $\varphi_{\mathcal{B}}(x) \leq \varphi_{\mathcal{D}}(y)$ for $x \in \mathcal{B}$ and $y \in \mathcal{D}$ if and only if there exists $\xi \in \mathcal{V}\left(V_{\mathcal{B}^{\approx}(x)}, V_{\mathcal{D}^{\approx}(y)}\right)$ such that $(x) \xi \tau_{y} \neq 0$.

In particular, if $\overline{\mathrm{El}}((\mathcal{B}, x),(\mathcal{D}, y)) \neq 0$, then $\varphi_{\mathcal{B}}(x) \leq \varphi_{\mathcal{D}}(y)$.

2. Let $s=\varphi_{\mathcal{B}}(x) \triangleleft t=\varphi_{\mathcal{D}}(y)$. Then there exists a morphism $\xi \in \mathcal{V}\left(V_{\mathcal{B}^{\approx}(x)}, V_{\mathcal{D} \approx(y)}\right)$ such that $x \xi=y$. Therefore, $0 \neq \overline{(0, \xi)} \in \overline{\mathrm{El}}((\mathcal{B}, x),(\mathcal{D}, y))$.

3. Let $s=\varphi_{\mathcal{B}}(x)=\varphi_{\mathcal{D}}(y)$ and let $s \nless s$. Let us prove that $\overline{\mathrm{El}}((\mathcal{B}, x),(\mathcal{D}, y)) \neq 0$ if $(\mathcal{B}, x) \leq(\mathcal{D}, y)$. By the definition of $\leq$, we have $\partial_{t}^{2}(\mathcal{B}, x) \leq \partial_{t}^{2}(\mathcal{D}, y)$ for every $t \in S^{\sim}(s)$. In particular, for an arbitrary nondegenerate vertex $x^{\prime} \in \Gamma^{-}(x)$, there is a unique nondegenerate vertex $y_{x^{\prime}}^{\prime} \in \Delta^{-}(y)$ such that $\varphi_{\mathcal{B}}\left(x^{\prime}\right)=\varphi_{\mathcal{D}}\left(y_{x^{\prime}}^{\prime}\right)$. We set $R_{x}=\left\{x^{\prime} \in \Gamma^{\sim}(x) \mid \partial_{x^{\prime}}^{2}(\mathcal{B}, x) \neq 1\right\}$.

By the induction hypothesis, we can assume that there exist nonzero morphisms $f_{x^{\prime}}=\overline{\left(y_{x^{\prime}}, \xi_{x^{\prime}}\right)} \in$ $\overline{\mathrm{E} l}\left(\partial_{x^{\prime}}^{2}(\mathcal{B}, x), \partial_{x^{\prime}}^{2}(\mathcal{D}, y)\right)$ defined for every $x^{\prime} \in R_{x}$. Let us decompose the vector spaces

$$
\begin{aligned}
& X_{\mathcal{B}}=V_{\mathcal{B}=(x)} \oplus\left(\underset{x^{\prime} \in R_{x}}{\oplus} X_{\partial_{x^{\prime}}^{2}(\mathcal{B}, x)}\right) \\
& X_{\mathcal{D}}=V_{\mathcal{D} \approx(y)} \oplus\left(\underset{x^{\prime} \in R_{x}}{\oplus} X_{\partial_{x^{\prime}}}^{2}(\mathcal{D}, y)\right) \oplus\left(\underset{y^{\prime} \in R_{y}, y^{\prime} \neq y_{x^{\prime}}^{\prime}}{\oplus} X_{\partial_{x^{\prime}}^{\prime}(\mathcal{D}, y)}^{2}\right) .
\end{aligned}
$$

Taking into account that $V_{\mathcal{B} \approx(x)}=V_{\mathscr{D} \approx(y)}$, we define the morphism $\xi$ as follows:

$$
\xi=\left(\begin{array}{ccc}
1_{V_{\mathcal{B}^{\approx}(x)}} & 0 & 0 \\
0 & \bigoplus_{x^{\prime} \in R_{x}} \xi_{x^{\prime}} & 0
\end{array}\right): X_{\mathcal{B}} \rightarrow X_{\mathcal{D}}
$$

We also define $\varphi: U_{\mathcal{B}} \rightarrow U_{\mathcal{D}}$ as a unique linear map such that $(\varphi, \xi) \in \operatorname{Rep} \mathcal{V}(R(\mathcal{B}), R(\mathcal{D}))$. One can directly verify that $0 \neq \overline{(\varphi, \bar{\xi})} \in \overline{\mathrm{El}}$. 
Assume that $\overline{\mathrm{El}}((\mathcal{B}, x),(\mathcal{D}, y)) \ni \overline{(\varphi, \xi)} \neq 0$. Let us prove that $(\mathcal{B}, x) \leq(\mathcal{D}, y)$, i.e., $\partial_{t}^{2}(\mathcal{B}, x) \leq \partial_{t}^{2}(\mathcal{D}, y)$ for every $t \in S^{\sim}(s)$. Let $x^{\prime} \in \mathcal{B}^{\approx}(x)$ and $y^{\prime} \in \mathcal{D}^{\approx}(y)$ denote vertices such that $\varphi_{\mathcal{B}}\left(x^{\prime}\right)=\varphi_{\mathcal{D}}\left(y^{\prime}\right)=t$; we can assume that $\partial_{t}^{2}(\mathcal{D}, y) \neq 1$. Furthermore, we set

$$
\alpha= \begin{cases}\bar{x}^{\prime} & \text { if } \Gamma^{-}\left(x^{\prime}\right)=\left\{\bar{x}^{\prime}\right\} \\ 0 & \text { if } \Gamma^{-}\left(x^{\prime}\right)=\varnothing\end{cases}
$$

Let $\bar{\xi} \in \mathcal{V}\left(V_{\mathcal{B}^{\approx}(x)}, V_{\mathcal{D}^{\approx}(y)}\right)$ be a component of the morphism $\xi$. Note that $\xi$ is invertible. If $\partial_{t}^{2}(\mathcal{D}, y)=0$, then $\tau_{y^{\prime}} \mid U_{\mathcal{D}}=0$. If $\partial_{t}^{2}(\mathcal{D}, y) \neq 0$, then $x^{\prime}+\alpha \in U_{\mathcal{B}}$ and $0 \neq\left(x^{\prime}+\alpha\right) \bar{\xi} \tau_{y^{\prime}}=\left(x^{\prime}+\alpha\right) \varphi \tau_{y^{\prime}}$. However, we have $\left(x^{\prime}+\alpha\right) \varphi \in U_{\mathcal{D}}$. Thus, we arrive at a contradiction. Therefore, $\partial_{i}^{2}(\mathcal{B}, x)=0$.

Consider the case $\partial_{t}^{2}(\mathcal{D}, y) \neq 0$. Let $\lambda$ be the component of the morphism $\xi$ that transforms $X_{\partial}^{2}(\mathcal{B}, x)$ into $X_{\partial_{t}{ }^{2}(\mathcal{D}, y)}$. Also assume that $\eta: U_{\partial_{t}{ }^{2}(\mathcal{B}, x)} \rightarrow X_{\partial_{t}{ }^{2}(\mathcal{D}, y)}$ is a uniquely defined linear map such that $(\eta, \lambda) \in$ $\mathrm{El}\left(\partial_{t}^{2}(\mathcal{B}, x), \partial_{t}^{2}(\mathcal{D}, y)\right)$. Let us prove that $\overline{(\eta, \lambda)} \neq 0$ in $\overline{\mathrm{El}}$.

For this purpose, it suffices to prove that $x^{\prime} \lambda \tau_{\bar{y}^{\prime}} \neq 0$. We have $0 \neq\left(x^{\prime}+\bar{x}^{\prime}\right) \xi \tau_{y^{\prime}}=\left(x^{\prime}+\bar{x}^{\prime}\right) \varphi \tau_{y^{\prime}}$. By virtue of step 1 of the proof and the fact that $\varphi_{\mathcal{D}}\left(y^{\prime}\right) \Varangle \varphi_{\mathcal{D}}\left(\bar{y}^{\prime}\right)$, the conditions $\beta \tau_{y^{\prime}} \neq 0$ and $\beta \tau_{\bar{y}^{\prime}} \neq 0$ are equivalent for $\beta \in U_{\mathcal{D}}$. This implies that $0 \neq\left(x^{\prime}+\bar{x}^{\prime}\right) \xi \tau_{\bar{y}^{\prime}}$. Furthermore, $\varphi_{\mathcal{B}}\left(x^{\prime}\right)=\varphi_{\mathcal{D}}\left(y^{\prime}\right) \nless \varphi_{\mathcal{D}}\left(\bar{y}^{\prime}\right)$ and, according to step 1 of the proof, $\left(x^{\prime}+\bar{x}^{\prime}\right) \xi \tau_{\bar{y}^{\prime}}=\bar{x}^{\prime} \xi \tau_{\bar{y}^{\prime}}$. Thus, $0 \neq \bar{x}^{\prime} \xi \tau_{\bar{y}^{\prime}}$ and $0 \neq \overline{(\eta, \lambda)} \in \overline{\mathrm{El}}$.

4. $s=\varphi_{\mathcal{B}}(x) \Rightarrow t=\varphi_{\mathfrak{D}}(y)$. The proof is similar to step 3; one should take into account that, in this case, there exists a morphism $\xi \in \mathcal{V}\left(V_{\mathcal{B}^{\approx}(x)}, V_{\mathcal{D}^{\approx}(y)}\right)$ of rank two such that $x \xi \tau_{y} \neq 0$.

Corollary 3. For a quasifinite vectroid $\mathcal{V}$, the relation $\leq$ on $C(\mathcal{V})$ is a partial ordering. The spectroid constructed on the basis of the poset $C(\mathcal{V})$ is isomorphic to the spectroid $\overline{\mathrm{El}}$.

Remark 9. One can also define the poset $C(\mathcal{V})$ for a chain vectroid $\mathcal{V}$ without assuming that $\operatorname{def} \mathcal{V} \leq 1$. In this case, the notions of $S(\mathcal{V})$-graphs and elementary representations remain the same, but, in item (d) of the definition of the ordering $\leq$ on $C(\mathcal{V})$, one must demand that the edges $s_{1} \Rightarrow s_{2}$ and $t_{1} \Rightarrow t_{2}$ be equivalent (see Remark 4).

\section{Multielementary Representations}

In this section, $\mathcal{V}$ denotes a chain vectroid. Let $\mathcal{U}$ be another chain vectroid. Then the completed biordered sets $S(\mathcal{U})$ and $S(\mathcal{V})$ and the set $C(\mathcal{V})$ of $S(\mathcal{V})$-graphs are given. Consider the map $C(\mathcal{V}) \rightarrow \hat{C}(\mathcal{V})$, $(\mathcal{B}, x) \mapsto \mathcal{B}$. Denote its composition with the map $R: \hat{C}(\mathcal{V}) \rightarrow \operatorname{Rep} \mathcal{V}$ by $P$.

Assume that the map $\lambda: S(\mathcal{U}) \rightarrow C(\mathcal{V})$ is given. Then the map of objects $\operatorname{Ex}(\lambda):$ Ob Rep $\mathcal{U} \rightarrow$ Ob Rep $\mathcal{V}$ is defined; it associates the representation $\operatorname{Ex}(\lambda) g$ of the vectroid $\mathcal{V}$ with the matrix

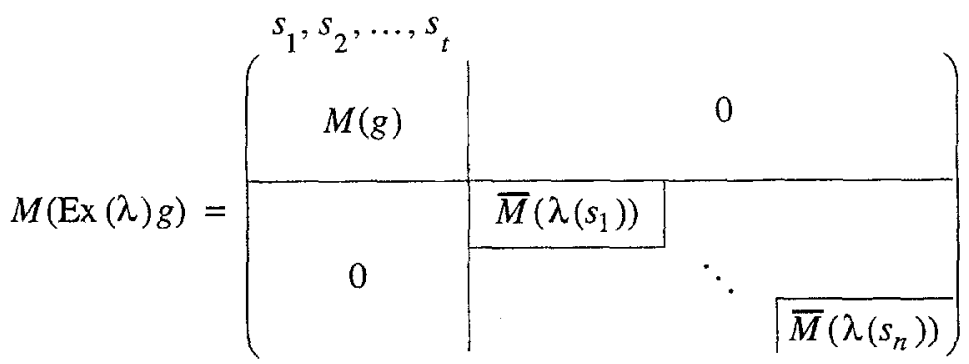


with the representation $(W, g, Z) \in \operatorname{Rep} \mathcal{U}$ with the matrix $M(g)$. Here, $\bar{M}\left(\lambda\left(s_{i}\right)\right)$ is the matrix of the elementary representation $P(\lambda(s))$ in which the (zero) column corresponding to the marked vertex $\lambda\left(s_{i}\right)$ of the $S(\mathcal{V})$ graph is omitted and replaced in the matrix $M(\operatorname{Ex}(\lambda) g)$ by the column of the matrix $M(g)$ marked by the element $s_{i}$.

Assume that the map $\lambda$ satisfies the following conditions:

(i) If $t_{1}, t_{2} \in S(\mathcal{U})$ and $t_{1} \approx t_{2}$, then $P \lambda\left(t_{1}\right) \approx P \lambda\left(t_{2}\right)$;

(ii) if $\lambda\left(t_{1}\right)<\lambda\left(t_{2}\right)$ (i.e., $\lambda\left(t_{1}\right) \neq \lambda\left(t_{2}\right)$ and $\overline{\mathrm{El}}\left(\lambda\left(t_{1}\right), \lambda\left(t_{2}\right)\right) \neq 0$ ), then $t_{1}<t_{2}$.

In this case, the representation $\operatorname{Ex}(\lambda) g$ is called the $\lambda$-extension of the representation $g$. In particular, let $\mathcal{V}$ be a quasifinite vectroid. Then the partial ordering $\leq$ is defined on $C(\mathcal{V})$ (see Sec. 5). Let $\mathcal{U}$ be a one-dimensional vectroid constructed on the basis of $C(\mathcal{V})$. For this vectroid, $S(\mathcal{U})=(C(\mathcal{V}), \leq, \leq, \Delta)$, where $\Delta$ is the diagonal. The image of the map $\mathrm{Mul}=\operatorname{Ex}\left(\mathrm{id}_{C(\mathcal{V})}\right): \mathrm{Ob} \operatorname{Rep} \mathcal{U}=\mathrm{Ob} \operatorname{Rep} C(\mathcal{V}) \rightarrow \mathrm{Ob} \operatorname{Rep} \mathcal{V}$ consists of representations of the vectroid $\mathcal{V}$ called multielementary representations.

Example 6 (Nonmultielementary representation). Let

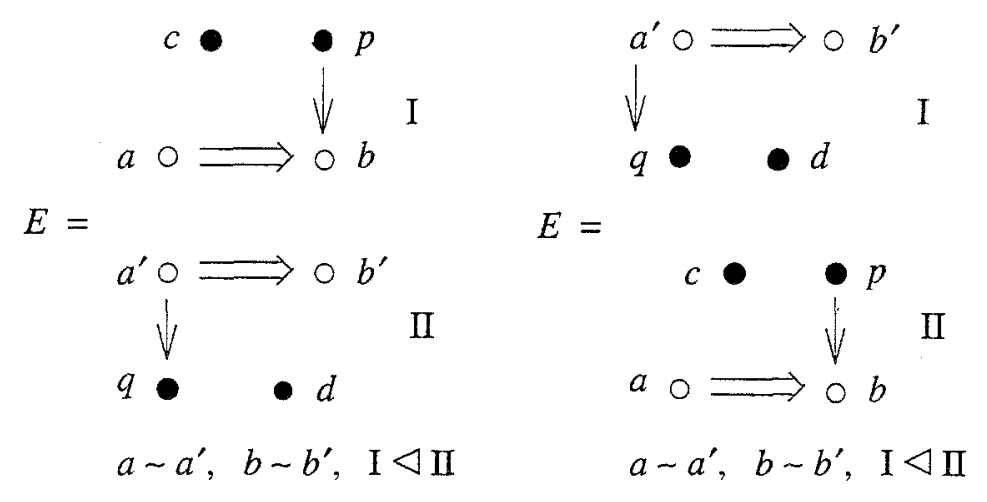

be completed biordered sets and let $\mathcal{E}=\operatorname{Vect}(E)$ and $\mathcal{E}^{*}=\operatorname{Vect}\left(E^{*}\right)$ (see Sec. 2). Then $E$ and $E^{*}$ can naturally be regarded as bipartite completed posets in the sense of $[11,12]$. It was proved in $[11,12]$ that $\mathcal{E}$ and $\mathcal{E}^{*}$ are finitely represented and each of them admits a faithful indecomposable representation unique to within an isomorphism, namely, the representation $g$ with the matrix

$$
M(g)=\left(\begin{array}{llllllll}
1 & 0 & 1 & 0 & 0 & 0 & 0 & 0 \\
1 & 0 & 0 & 1 & 0 & 0 & 0 & 0 \\
0 & 0 & 0 & 0 & 1 & 1 & 0 & 0 \\
0 & 0 & 0 & 0 & 1 & 0 & 1 & 1
\end{array}\right)
$$

The columns of this matrix correspond to $a, b, c, p, a^{\prime}, b^{\prime}, d, q$. The representation $g$ is not multielementary. It is called the exceptional representation (of $E$ or $E^{*}$ ).

Proposition 6. Let $\mathcal{V}$ be a quasifinite vectroid and let $g_{1}$ and $g_{2}$ be representations of the poset $C(\mathcal{V})$. Then the following assertions are true: 
(i) $\operatorname{Mul}\left(g_{1} \oplus g_{2}\right) \simeq \operatorname{Mul}\left(g_{1}\right) \oplus \operatorname{Mul}\left(g_{2}\right)$;

(ii) if $g_{1} \simeq g_{1}[$ in $\operatorname{Rep} C(\mathcal{V})]$, then $\operatorname{Mul}\left(g_{1}\right) \simeq \operatorname{Mul}\left(g_{2}\right)($ in $\operatorname{Rep} \mathcal{V})$.

To prove the proposition we perform the following construction: Let $(A, M) \in \mathcal{M}$ be a module over the aggregate and let $\operatorname{Der}(A, M)=\left(\operatorname{Rep} M, E_{M}\right)$ be a derivative module. Assume that another module $(B, N) \in \mathcal{M}$ over the aggregate and the morphism $(F, \Phi):(B, N) \rightarrow \operatorname{Der}(A, M) \in \operatorname{Mor} \mathcal{M}$ are given. In this case, we can define a morphism $(I, \Pi): \operatorname{Der}(B, N) \rightarrow \operatorname{Der}(A, M)$. Here, $I: \operatorname{Rep} N \rightarrow \operatorname{Rep} M$ is the functor that takes the value

$$
I(W, g, Z)=\left(W \oplus V,\left(\begin{array}{c}
g \circ \Phi(Z) \\
f
\end{array}\right), X\right)
$$

on the representation $(W, g, Z) \in \operatorname{Rep} N$ with $F(Z)=(V, f, X)$, and $\Pi: E_{N} \rightarrow I^{*} E_{M}$ is the homomorphism of $B$-modules defined by the following diagram:

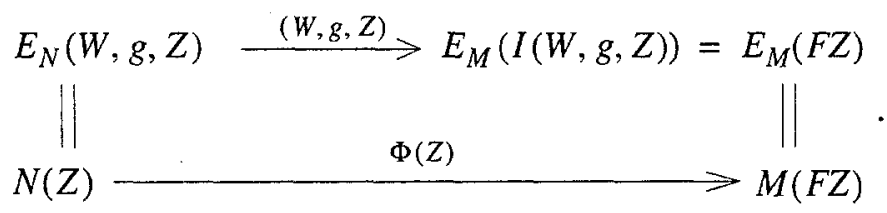

Under certain additional conditions, which can easily be written in the explicit form, $I(W, g, Z)$ is the $\lambda$-extension of the representation $g$.

Proof of Proposition 6. Assume that $(B, N)$ is a module over the aggregate obtained from the module over the spectroid $(\mathrm{El}, N)$, i.e., $B=\oplus \mathrm{El}$ (see $\operatorname{Sec} .5$ ). We define a morphism $(F, \Phi):(B, N) \rightarrow \operatorname{Der}(\mathcal{V})$, setting $F(\mathcal{B}, x)=R(\mathcal{B})$, as follows:

$$
\begin{gathered}
\Phi(\mathcal{B}, x): N(\mathcal{B}, x)=a_{\mathcal{B}, x} k \rightarrow \mathcal{X}_{\mathcal{B}}=E_{\mathcal{V}}\left(U_{\mathcal{B}}, i_{\mathcal{B}}, X_{\mathcal{B}}\right), \\
a_{\mathcal{B}, x} \Phi(\mathcal{B}, x)=x \in X_{\mathcal{B}} .
\end{gathered}
$$

By applying the construction described above, we obtain a functor $I: \operatorname{Rep} N \rightarrow \operatorname{Rep} \mathcal{V}$. On the other hand, the category $\operatorname{Rep} N$ is naturally epivalent to the category $\operatorname{Rep} C(\mathcal{V})$ (see Corollary 3 ). It is easy to see that every preimage $f$ of a representation $g \in C(\mathcal{V})$ in $\operatorname{Rep} N$ has the same matrix as $g$, and $I(f)$ is the representation of $\operatorname{Mul}(g)$. This obviously proves Proposition 6.

If $\operatorname{dim} \mathcal{V}=2$, then the poset $C(\mathcal{V})$ coincides (by definition) with the poset of "flaggened sequences" $\mathrm{St}(\mathcal{V})$ defined in $([1], 5.8)$. It was proved in [7-9] that a vectroid $\mathcal{V}$ of dimension $\leq 2$ is finitely represented if and only if the poset $C(\mathcal{V})$ is finitely represented. However, in this case, not all indecomposable representations are multielementary $[11,12]$.

Hypothesis 1. A vectroid $\mathcal{V}$ is finitely represented if and only if $\mathcal{V}$ is a chain vectroid, def $\mathcal{V} \leq 1$, $\operatorname{dim} \mathcal{V} \leq 3$, and the poset $C(\mathcal{V})$ is finitely represented.

Hypothesis 2. Suppose that a vectroid $\mathcal{V}$ is finitely represented and $g$ is its faithful representation. Then $g$ satisfies one of the following conditions: 
(i) $g$ is decomposable;

(ii) $g$ is indecomposable and multielementary;

(iii) $g$ is the $\lambda$-extension of the exceptional representation of $E$ or $E^{*}$ for proper $\lambda: E \rightarrow C(\mathcal{V})$ or $\lambda: E^{*} \rightarrow C(\mathcal{V})$ (in particular, $g$ is indecomposable).

The authors are indebted to P. Gabriel for discussions, in which the idea of this paper emerged, and numerous remarks taken into account in the final version of the paper.

This work was supported by the Foundation for Fundamental Research of the Ukrainian State Committee on Science and Technology and the International Science Foundation (Grant No. U6E000).

\section{REFERENCES}

1. P. Gabriel and A. V. Roiter, "Representations of finite-dimensional algebras," in: Encyclopaedia of Mathematical Sciences, Vol. 73, Algebra 8, Springer-Verlag, New York (1992).

2. L. A. Nazarova and A. V. Roiter, Category Matrix Problems and the Brauer-Thrall Problem [in Russian], Preprint No. 09.73, Institute of Mathematics, Ukrainian Academy of Sciences, Kiev (1973).

3. P. Gabriel, L. A. Nazarova, A. V. Roiter, V. V. Sergeichuk, and D. Vossieck, "Tame and wild subspace problems," Ukr. Mat. Zh., 46, No. 3, 313-352 (1993).

4. A. V. Roiter and V. V. Sergeichuk, "Existence of a multiplicative basis for a finitely spaced module over an aggregate," Ukr. Mat. Zh., 46, No. 5, 567-579 (1994).

5. L. A. Nazarova and A. V. Roiter, "Representations of partially ordered sets," Zap. Nauchn. Sem. Leningrad. Otd. Mat. Inst. Akad. Nauk SSSR, 28, 5-31 (1972).

6. M. M. Kleiner, "Partially ordered sets of finite type," Zap. Nauchn. Sem. Leningrad. Otd. Mat. Inst. Akad. Nauk SSSR, 28, 32-41 (1972).

7. L. A. Nazarova and A. V. Roiter, Representations of Biinvolutive Partially Ordered Sets [in Russian], Preprint No. 91.34 , Institute of Mathematics, Ukrainian Academy of Sciences, Kiev (1991).

8. L. A. Nazarova and A. V. Roiter, Representations of Biinvolutive Partially Ordered Sets. II [in Russian], Preprint No. 94.02 , Institute of Mathematics, Ukrainian Academy of Sciences, Kiev (1994).

9. L. A. Nazarova and A. V. Roiter, "Representations and forms of weakly completed partially ordered sets," in: Linear Algebra and Representation Theory [in Russian], Institute of Mathematics, Ukrainian Academy of Sciences, Kiev (1983), pp. 19-54.

10. D. Vossieck, "Représentations de bifoncteurs et interprétation en termes de modules," Comptes-Rendus Acad. Sc. Paris., 307, 713-716 (1988).

11. L. A. Nazarova and A. V. Roiter, Representations of Bipartite Completed Sets [in Russian], Preprint No. 87.05, Institute of Mathematics, Ukrainian Academy of Sciences, Kiev (1987).

12. L. A. Nazarova and A. V. Roiter, "Representations of bipartite completed posets," Comment. Helv. Math., 63, 498-526 (1988).

13. G. Birkhoff, Lattice Theory, Providence, Rhode Island (1967).

14. K. Kuratowski and A. Mostowski, Theory of Sets [Russian translation], Mir, Moscow (1970). 